Check for updates

Cite this: RSC Adv., 2017, 7, 31807

\title{
Cudrania tricuspidata: an updated review on ethnomedicine, phytochemistry and pharmacology
}

Received 17th April 2017

Accepted 3rd June 2017

DOI: $10.1039 / \mathrm{c} 7 \mathrm{ra0} 4322 \mathrm{~h}$

rsc.li/rsc-advances

\author{
Lan-Ting Xin, (D) † $^{\mathrm{ab}}$ Shi-Jun Yue, $\dagger^{\mathrm{ab}}$ Ya-Chu Fan, ${ }^{\mathrm{ab}}$ Jing-Shuai Wu, ${ }^{\text {ab }}$ Dan Yan, ${ }^{\mathrm{c}}$ \\ Hua-Shi Guan*ab and Chang-Yun Wang (D) *ab
}

Cudrania tricuspidata is a perennial plant of the family Moraceae with numerous medicinal and nutritional applications. It has been widely used in East Asia as an important traditional folk medicine for the treatment of many ailments such as eczema, mumps, tuberculosis, contusions, insomnia and acute arthritis. The whole plant of $C$. tricuspidata, including the roots, leaves, bark, stems and fruits, has been found to contain diverse phytochemicals, including xanthones, flavonoids, organic acids, and polysaccharides, with various bioactivities. In particular, xanthones and flavonoids, as the main active constituents, isolated from C. tricuspidata have been proven to possess notable anti-inflammatory, antioxidative, antitumor, hepatoprotective, neuroprotective and anti-obesity effects. This review summarizes the botany, traditional uses, phytochemistry and pharmacology of C. tricuspidata, and the limitations of studies on this species have also been discussed such that to serve as the basis for further research and development on this medicinal plant.

\section{Introduction}

Cudrania tricuspidata (Carr.) Bur. ex Lavallee, which is a deciduous thorny tree belonging to the family Moraceae, is widespread throughout East Asia $^{\mathbf{1}}$ and is known as cudrang, mandarin melon berry, silkworm thorn, storehouse bush, hariguwa (in Japanese) and che (in Chinese). ${ }^{2-4}$ In China, C. tricuspidata roots have been used as 'Chuan-po-shi' in traditional Chinese medicine (TCM) in the treatment of gonorrhea, rheumatism, jaundice, boils, scabies, bruising, and dysmenorrhea; ${ }^{5,6}$ its root bark has been widely used in the treatment of lumbago, hemoptysis, and contusions; ${ }^{7}$ and its roots and stems have also been applied in the forms of syrups, granules and injections to cure tumors of the digestive tract. ${ }^{8}$ In Korea, $C$. tricuspidata has become one of the most ubiquitous folk remedies against cancer during the last few decades. ${ }^{9}$ In addition to the above medical uses, in China the stems and roots of C. tricuspidata have been used to prepare herbal teas or functional beverages for a long period; ${ }^{10}$ the stems, which contain a reddish-yellow dye, have been noted for their use in coloring imperial garments; ${ }^{3}$ and the tender leaves of $C$. tricuspidata have been used as a perfect food for breeding silkworms since the

${ }^{a}$ Key Laboratory of Marine Drugs, The Ministry of Education of China, School of Medicine and Pharmacy, Ocean University of China, Qingdao 266003, P. R. China. E-mail: changyun@ouc.edu.cn; hsguan@ouc.edu.cn; Fax: +86 532 82031536; Tel: +8653282031536; +8653282031667

${ }^{b}$ Laboratory for Marine Drugs and Bioproducts, Qingdao National Laboratory for Marine Science and Technology, Qingdao 266071, P. R. China

${ }^{c}$ Beijing Shijitan Hospital, Capital Medical University, Beijing 100038, P. R. China

$\dagger$ These authors have contributed equally to this work.
Han dynasty, and the natural silk has been praised under the name of Zhe $\mathrm{Si}^{11}{ }^{11}$ Its edible fruits have been made into juices, jams, alcoholic beverages, dietary supplements and other health products in Korea. ${ }^{12}$ Moreover, its bark fibers have been utilized to make paper and its trunks have been used as valuable timber for furniture manufacture. ${ }^{13}$

The immense medicinal and economic value of C. tricuspidata has encouraged numerous studies of its phytochemicals and pharmacological activities. C. tricuspidata extracts have been demonstrated to possess good therapeutic effects against various ailments including inflammation, ${ }^{14,15}$ tumors, ${ }^{16,17}$ obesity, ${ }^{\mathbf{1 8 , 1 9}}$ and diabetes. ${ }^{\mathbf{2 0 , 2 1}}$ Xanthones and flavonoids have been considered to be the two major classes of phytochemicals in C. tricuspidata. For example, prenylated xanthones and flavonoids were found to be the most important and abundant constituents in its leaves and root bark with regard to their notable anti-inflammatory, ${ }^{22,23}$ antitumor, ${ }^{16,24}$ hepatoprotective,,$^{25,26}$ neuroprotective,,$^{27,28}$ and anticoagulant ${ }^{29}$ activities; hydroxybenzyl flavonoid glycosides from the stem bark were reported to be promising natural antioxidant and antitumor agents; ${ }^{30}$ and prenylated isoflavonoids and benzylated flavonoids from the fruits displayed potential antiinflammatory $^{31}$ and antioxidant ${ }^{32}$ activities. Besides, a glycoprotein $(75 \mathrm{kDa})$ from $C$. tricuspidata, which consisted of carbohydrate $(72.5 \%)$ and protein moieties $(27.5 \%)$, exhibited distinctive characteristics with anti-inflammatory, ${ }^{33}$ antioxidant,$^{34}$ hepatoprotective, ${ }^{35}$ and immunomodulatory ${ }^{36}$ effects.

To date, to the best of our knowledge, no comprehensive review concerning $C$. tricuspidata has been available. A literature survey was conducted via an electronic search using PubMed, 
Scopus, ACS, Web of Science, ScienceDirect, China Knowledge Resource Integrated Database (CNKI), Google Scholar, SciFinder and a library search for ethnobotanical textbooks. The Plant List (www.theplantlist.org), the Missouri Botanical Garden's Tropicos nomenclature database (www.tropicos.org) and the Chinese Field Herbarium (www.cfh.ac.cn) were used to validate the taxonomy and also obtain information regarding subspecies and cultivars. On the basis of the literature search, we reviewed the achievements of research on the botanical characteristics, traditional uses, phytochemicals and pharmacological activities of $C$. tricuspidata so as to provide a systematic summary of the literature for further research on, and development of, this medicinal plant.

\section{Botany and traditional uses of $C$. tricuspidata}

\subsection{Botany of $C$. tricuspidata}

C. tricuspidata is one of six species in the genus Cudrania, which is endemic to Asia and Oceania, of the family Moraceae in the order Urticales. $^{37}$ Five species, namely, C. tricuspidata, $C$. cochinchinensis (Lour.) Kudo et Masam, C. fruticosa (Roxb.) Wight ex Kurz, C. amboinensis (Bl.) Miq., and C. pubescens Trec., as well as $C$. cochinchinensis var. gerontogea (Nakai) Kudo et Masam, have been widely cultivated in Southern China, including Yunnan, Fujian and Jiangsu provinces, as well as in Hebei province, as profitable plants for producing valuable fruits and timber. ${ }^{38,39}$ In Europe $C$. tricuspidata was introduced into cultivation in 1870 , and in the USA in $1909 .{ }^{40}$ In contrast, wild populations of this species are now under threat of extinction.

As a hardy deciduous plant, C. tricuspidata is widely distributed in lowlands, foothills, forests, or dense scrub at altitudes of between 500 and $2000 \mathrm{~m}$. It can eventually grow to a height of approximately 1.0-7.0 m (Fig. 1), but often exists as a broad spreading bush or small tree. Its leaves have single alternate ovate to rhombic-ovate blades with a size of 5.0$14.0 \mathrm{~cm}$ long and 3.0-6.0 $\mathrm{cm}$ wide; its flowers have a dioecious capitulum with an inflorescence length of 0.5 (male) or 1.0$1.5 \mathrm{~cm}$ (female); the color of its syncarpous fruits is orange-red when mature; and its roots, which are up to $50 \mathrm{~cm}$ long, are yellow and irregularly cylindrical. ${ }^{37}$

\subsection{Traditional uses}

Since ancient times, $C$. tricuspidata has been used as a folk medicine in oriental countries. ${ }^{41}$ The medicinal material, which is known as Zhemu in TCM, is sweet in taste, slightly warm in nature and acts on the liver and spleen meridians in TCM theory. ${ }^{42}$ Its medicinal usage was first documented in Ben Cao Shi Yi (700-800 A.D., Tang Dynasty), which is a famous masterpiece of TCM ${ }^{42}$ followed by other Chinese medical classics such as Ben Cao Yan Yi (1116 A.D., Song Dynasty), ${ }^{43}$ Ben Cao Hui Yan (1624 A.D., Ming Dynasty), ${ }^{44}$ and the Dictionary of Chinese Herbal Medicine (2006 Edition). ${ }^{6}$ Notably, C. tricuspidata roots, together with the roots of $C$. cochinchinensis (Lour.) Kudo et Masam, have been recorded as 'Chuan-po-shi' in the Chinese

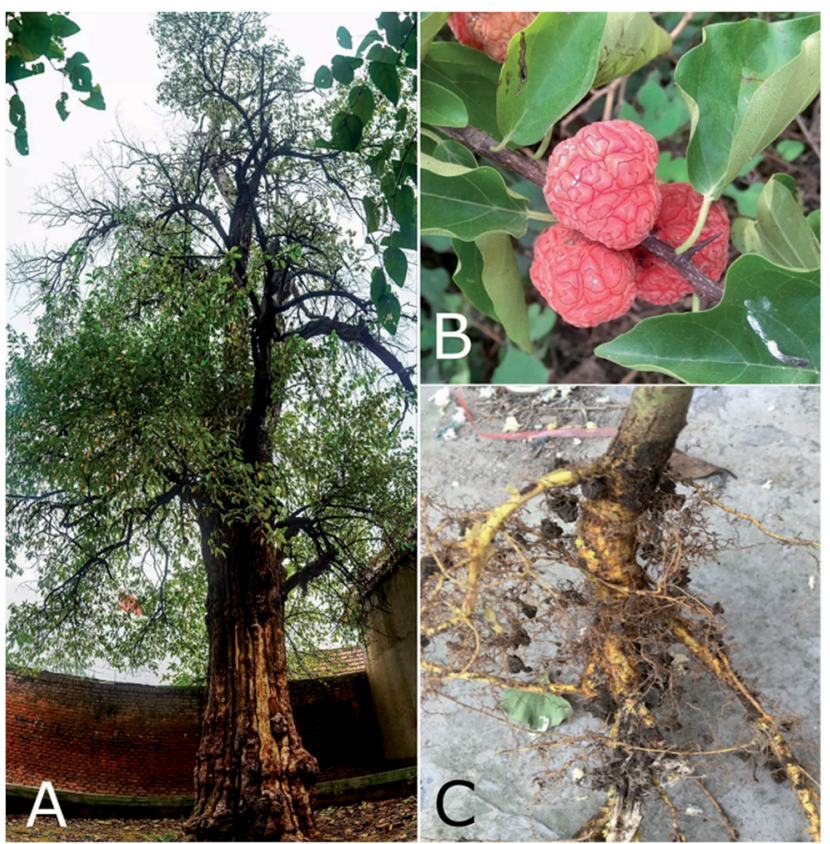

Fig. 1 Whole plant ( 200 years) (A), fruits (B) and roots (C) of $C$. tricuspidata.

Pharmacopoeia (1977 Edition). ${ }^{5}$ According to traditional applications and empirical practice, the bark of $C$. tricuspidata has mainly been used to strengthen the body and improve health conditions, and the trunk to invigorate the circulation of the blood and cure impaludism. ${ }^{42}$ An aqueous decoction of its fruits and leaves (15-30 g) could be taken orally to relieve rheumatoid arthritis. Scabies and eczema could be alleviated using a decoction of its roots mixed with the roots of $C$. cochinchinensis and glutinous rice. ${ }^{45}$ An aqueous decoction of its roots combined with Acanthus ilicifolius L. and Desmodium pulchellum (L.) Benth. was documented to treat hepatitis, in particular viral hepatitis. ${ }^{\mathbf{4 6 7}}$ Currently, the bark of $C$. tricuspidata, together with Zhemu syrup (a traditional Chinese patent medicine), is widely employed in TCM clinics for the treatment of cancer of the alimentary system, in particular gastric carcinoma. ${ }^{\mathbf{8 2 4}}$ Specifically, $C$. tricuspidata has been widely used with a long folkloric medicinal history by Chinese nationalities including Yi, Wa, Tong, Bai and Yao.

In the Korean classic Donguibogam (1613 A.D., Joseon Dynasty), C. tricuspidata was recorded as treating eczema, mumps, tuberculosis, contusions, and acute arthritis. ${ }^{48}$ Its fruits are commonly consumed in the Korean daily diet owing to its diverse biological effects, e.g., antioxidant, antiinflammatory and immunomodulatory activities. ${ }^{12}$ In addition, during the last few decades, the whole plant of C. tricuspidata has been exploited as an important folk remedy for cancer in Korea. ${ }^{49}$

\section{Phytochemistry}

Over recent decades, a large number of chemical constituents have been isolated from $C$. tricuspidata. Xanthones and 
flavonoids have been recognized to be the main active and structurally diverse constituents responsible for the various activities of this species, followed by organic acids, polysaccharides, phenylpropanoids, and other constituents (Table 1).

\subsection{Xanthones (1-99)}

The presence of abundant xanthones substituted by a variety of isoprenoid, phenolic and methoxy groups has been considered to be a taxonomic feature of C. tricuspidata (Fig. 2)..$^{17,49,50}$ Among these, from the perspective of the structure-activity relationship (SAR), xanthones substituted by isoprenoid groups display better biological activities. For instance, cudratricusxanthone A (CTXA, 1), cudraxanthones L (28) and M (29), and macluraxanthone B (72) are notable isoprenylated xanthones with antiinflammatory, ${ }^{22}$ antitumor, ${ }^{17}$ neuroprotective, ${ }^{51}$ hepatoprotective, ${ }^{26}$ monoamine oxidase (MAO)-inhibiting, ${ }^{50}$ anticoagulant, ${ }^{29}$ antidiabetic, ${ }^{52}$ and neuraminidase-inhibiting effects. ${ }^{53}$ Cudratricusxanthones B-E (2-5) and G (7), cudraxanthones D (20), L and M, and macluraxanthones B and C (73), which are classed as catecholic xanthones, could be converted into quinone methide intermediates in an enzymatic or a nonenzymatic manner ${ }^{54}$ and were reported to have significant antitumor activity. ${ }^{17,49,55,56}$ In addition, catecholic xanthones, specifically cudraxanthone C (19) and 1,3,7-trihydroxy-4-(1,1dimethyl-2-propenyl)-5,6-(2,2-dimethylchromeno)xanthone (84), exhibited both potent superoxide- and hydroxyl radicalscavenging activities, which could be rationalized by their chelating effect with $\mathrm{Fe}^{2+}$ ions. ${ }^{56}$

It should be noted that investigations into quantitative analysis of the characteristic xanthones in C. tricuspidata are scarce. On the basis of HPLC analysis, cudratricusxanthones B, $\mathrm{D}$ and $\mathrm{F}(\mathbf{6})$ and macluraxanthone $\mathrm{B}$ in C. tricuspidata root bark were found to account for $0.017 \%, 0.026 \%, 0.025 \%$ and $0.071 \%$, respectively. ${ }^{57}$ The quantitative analysis of other xanthones in $C$. tricuspidata is worth investigating in the future.

\subsection{Flavonoids (100-257)}

Flavonoids account for the largest proportion of C. tricuspidata and have attracted particular interest because of their welldefined pharmacological activities. To date, more than 120 flavonoids (Fig. 2) have been isolated from C. tricuspidata and can be classified into flavones (100-128), flavanones (129-173), and isoflavones (174-257). Structurally, the majority of them possess prenylated, benzylated, and methoxy groups substituted on their aromatic rings. Cudraflavanone E (133), which is isolated from C. tricuspidata roots, features a rare flavanone skeleton with the B-ring fused to a furan ring. ${ }^{58}$ Lee et al. ${ }^{16,59}$ isolated a series of rare benzyl-substituted flavonoids, i.e., gericudranins A-E (161-165), from C. tricuspidata. Notably, prenylated flavonoids have been regarded as attractive specialized metabolites with diverse biological activities. Specifically, cudraflavone B (104), which is a prominent prenylated flavonoid from the roots of C. tricuspidata, exhibited MAO-inhibiting, ${ }^{50}$ antiatherosclerotic, ${ }^{9}$ anti-inflammatory, ${ }^{60}$ hepatoprotective, ${ }^{25}$ antitumor, ${ }^{61}$ and neuroprotective ${ }^{62}$ effects. Euchrestaflavanones
B (143) and C (144) displayed antibacterial activity against Gram-positive bacteria, Staphylococcus aureus, Bacillus subtilis and Bacillus cereus. ${ }^{63}$

The investigation of the biosynthesis of the prenylflavonoids in C. tricuspidata has been attempted. Dai et al. ${ }^{64}$ established a cell suspension culture of $C$. tricuspidata for the enzymatic preparation of prenylflavonoids. A flavonoid prenyltransferase was identified as C. tricuspidata isoliquiritigenin $3^{\prime}$-dimethylallyltransferase. ${ }^{64}$ This enzyme was found to be able to regioselectively introduce dimethylallyl diphosphate at the orthoposition of the phenolic moiety in the common 2,4-dihydroxyacetophenone substructure shared by the three types of flavonoids, i.e., chalcones, isoflavones, and flavones. ${ }^{64,65}$ These studies could improve our knowledge of the mechanism of the biosynthesis and accumulation of prenylated flavonoids in $C$. tricuspidata.

The constituents and quantities of the flavonoids in C. tricuspidata fruits have been demonstrated to change in accordance with their maturation stage. Unripe fruits of $C$. tricuspidata were found to have a higher content of total flavonoids in comparison with ripe fruits. An analysis of the chemical constituents revealed that flavonoids with a side chain of cyclized prenyl 2,2-dimethylpyran rings were predominant in the unripe fruits, whereas flavonoids with a linear prenyl side chain were the main constituents in ripe fruits. ${ }^{66}$

Only a few studies have been reported concerning the quantitative analysis of representative flavonoids in C. tricuspidata. By UV-vis spectrophotometry, the concentration of total flavonoid glycosides in C. tricuspidata roots was measured to be up to $3.96 \mathrm{mg} \mathrm{g}^{-1}$ (as rutin equivalents). ${ }^{67}$ By HPLC analysis, the flavonoids kaempferol (118), quercetin (125), naringenin (146) and taxifolin (166) in C. tricuspidata were found to occur at 0.30 , $0.09,1.94$ and $0.63 \mathrm{mg} \mathrm{g}^{-1}$ in the roots and $0.08,0.04,0.90$ and $0.62 \mathrm{mg} \mathrm{g}^{-1}$ in the stems, respectively. ${ }^{68}$ Jeon et al..$^{69}$ reported the isolation of prenylated isoflavonoids from an $n$-hexane extract of C. tricuspidata fruits using centrifugal partition chromatography and found that the main flavonoids $4^{\prime}-O$-methylalpinumisoflavone (242), 6,8-diprenylgenistein (252) and 6,8-diprenylorobol (254) amounted to $2.7 \%, 7.6 \%$ and $6.4 \%$, respectively.

\subsection{Organic acids (258-289)}

To date, thirty-one organic acids and their esters have been isolated from $C$. tricuspidata. The stem extract was reported to contain $n$-hexanoic acid (278) in the greatest concentration (9.89 $\left.\mu \mathrm{g} \mathrm{g}^{-1}\right)$ followed by 2-acetylpyrrole $\left(405,1.86 \mu \mathrm{g} \mathrm{g}^{-1}\right)$, whereas the root extract was found to have $n$-hexanoic acid $\left(13.13 \mu \mathrm{g} \mathrm{g}^{-1}\right)$ in the greatest concentration followed by $n$-heptanoic acid (279, $\left.2.05 \mu \mathrm{g} \mathrm{g}^{-1}\right) .{ }^{10}$ Jung et al. ${ }^{70}$ reported that the levels of organic acids such as citric acid (261), malic acid (264), oxalic acid (265), succinic acid (270) and tartaric acid (273) in C. tricuspidata fruits varied with the maturation stage, and malic acid was the most abundant.

\subsection{Polysaccharides (290-295)}

The yield of total polysaccharides (CTPS) amounted to $1.0 \%$ in the roots of $C$. tricuspidata. ${ }^{71}$ Six polysaccharides with strong 
Table 1 Chemical constituents of $C$. tricuspidata

No. Compound name(s) Tissue(s) Ref.

Xanthones

Cudratricusxanthone A

Cudratricusxanthone B

Cudratricusxanthone $\mathrm{C}$

Cudratricusxanthone D

Cudratricusxanthone E

Cudratricusxanthone $\mathrm{F}$

Cudratricusxanthone $\mathrm{G}$

Cudratricusxanthone $\mathrm{H}$

Cudratricusxanthone I

Cudratricusxanthone J

Cudratricusxanthone $\mathrm{K}$

Cudratricusxanthone $\mathrm{L}$

Cudratricusxanthone $\mathrm{M}$

Cudratricusxanthone $\mathrm{N}$

Cudratricusxanthone $\mathrm{O}$

Cudratricusxanthone P/cudracuspixanthone A

Cudraxanthone A

Cudraxanthone B

Cudraxanthone $\mathrm{C}$

Cudraxanthone D

Cudraxanthone E

Cudraxanthone $\mathrm{F}$

Cudraxanthone $\mathrm{G}$

Cudraxanthone $\mathrm{H}$

Cudraxanthone I

Cudraxanthone J

Cudraxanthone $\mathrm{K}$

Cudraxanthone L

Cudraxanthone $\mathrm{M}$

Cudraxanthone $\mathrm{N}$

Cudraxanthone $\mathrm{O}$

Cudracuspixanthone $\mathrm{B} /$ cudratrixanthone $\mathrm{B}$

Cudracuspixanthone $\mathrm{C}$

Cudracuspixanthone D

Cudracuspixanthone E

Cudracuspixanthone $\mathrm{F}$

Cudracuspixanthone $\mathrm{G}$

Cudratrixanthone A

Cudratrixanthone C

Cudratrixanthone D

Cudratrixanthone E

Cudratrixanthone $\mathrm{F}$

Cudratrixanthone G

Cudratrixanthone $\mathrm{H}$

Cudratrixanthone I

Cudratrixanthone J

Cudratrixanthone $\mathrm{K}$

Cudratrixanthone $\mathrm{L}$

Cudratrixanthone $\mathrm{M}$

Cudratrixanthone $\mathrm{N}$

Cudratrixanthone $\mathrm{O}$

Cudratrixanthone $\mathrm{P}$

Cudratrixanthone Q

Cudratrixanthone $\mathrm{R}$

Cudratrixanthone $\mathrm{S}$

Cudratrixanthone $\mathrm{T}$

Cudratrixanthone U

Cudratrixanthone $\mathrm{V}$

Cudratrixanthone $\mathrm{W}$

Alvaxanthone

Alloathyriol

Dulxanthone B

\begin{tabular}{|c|c|}
\hline Whole plant & 49 \\
\hline Roots & 17 \\
\hline Roots & 17 \\
\hline Roots & 17 \\
\hline Roots & 17 \\
\hline Roots & 17 \\
\hline Roots & 17 \\
\hline Roots & 17 \\
\hline Roots & 55 \\
\hline Roots & 50 \\
\hline Roots & 50 \\
\hline Roots & 50 \\
\hline Roots & 50 \\
\hline Roots & 143 \\
\hline Roots & 143 \\
\hline Roots & 116 and 143 \\
\hline Root bark & 144 \\
\hline Root bark & 144 \\
\hline Root bark & 144 \\
\hline Root bark & 4 \\
\hline Root bark & 146 \\
\hline Root bark & 146 \\
\hline Root bark & 146 \\
\hline Root bark & 147 \\
\hline Root bark & 147 \\
\hline Root bark & 147 \\
\hline Root bark & 147 \\
\hline Root bark & 148 \\
\hline Root bark & 148 \\
\hline Root bark & 148 \\
\hline Root bark & 148 \\
\hline Roots & 27 and 116 \\
\hline Roots & 116 \\
\hline Roots & 116 \\
\hline Roots & 88 \\
\hline Roots & 88 \\
\hline Roots & 88 \\
\hline Root bark & 27 \\
\hline Root bark & 27 \\
\hline Root bark & 27 \\
\hline Root bark & 27 \\
\hline Root bark & 27 \\
\hline Root bark & 27 \\
\hline Root bark & 27 \\
\hline Root bark & 27 \\
\hline Root bark & 27 \\
\hline Root bark & 27 \\
\hline Root bark & 27 \\
\hline Root bark & 27 \\
\hline Root bark & 27 \\
\hline Root bark & 27 \\
\hline Root bark & 145 \\
\hline Root bark & 145 \\
\hline Root bark & 145 \\
\hline Root bark & 145 \\
\hline Root bark & 145 \\
\hline Root bark & 145 \\
\hline Root bark & 145 \\
\hline Root bark & 145 \\
\hline Roots & 88 \\
\hline Roots & 88 \\
\hline Twigs & 75 \\
\hline
\end{tabular}


Table 1 (Contd.)

\begin{tabular}{|c|c|c|c|}
\hline No. & Compound name(s) & Tissue(s) & Ref. \\
\hline 63 & Gerontoxanthone A & Root bark & 25 \\
\hline 64 & Gerontoxanthone C & Root bark & 27 \\
\hline 66 & Isocudraniaxanthone A & Roots & 116 \\
\hline 67 & Isocudraniaxanthone B & Root bark & 56 \\
\hline 68 & Isocudraxanthone $\mathrm{K}$ & Root bark & 25 \\
\hline 71 & Laxanthone I & Root bark & 116 \\
\hline 72 & Macluraxanthone B & Whole plant & 49 \\
\hline 73 & Macluraxanthone C & Roots & 55 \\
\hline 74 & Nigrolineaxanthone F & Root bark & 27 \\
\hline 75 & Neriifolone A & Root bark & 27 \\
\hline 76 & Toxyloxanthone B & Root bark & 145 \\
\hline 81 & 1,3,5-Trihydroxy-2-(3-methylbut-2-enyl)xanthone & Root bark & 27 \\
\hline 82 & 1,3,5,6-Tetrahydroxyxanthone & Bark & 149 \\
\hline 83 & $\begin{array}{l}\text { 1,3,6,7-Tetrahydroxy-2-(3-methylbut-2-enyl)- } \\
\text { 8-(2-methylbut-3-en-2-yl)-9H-xanthen-9-one }\end{array}$ & Roots & 52 \\
\hline 84 & $\begin{array}{l}\text { 1,3,7-Trihydroxy-4-(1,1-dimethyl-2-propenyl)- } \\
\text { 5,6-(2,2-dimethylchromeno)xanthone }\end{array}$ & Roots & 52 \\
\hline 85 & 1,5-Dihydroxy-3,6-dimethoxyxanthen-9-one & Twigs & 75 \\
\hline 86 & 1,7-Dihydroxy-3,6-dimethoxyxanthone & Roots & 55 \\
\hline 87 & $\begin{array}{l}\text { 1,6,7-Trihydroxy-2-(1,1-dimethyl-2-propenyl)- } \\
\text { 3-methoxyxanthone }\end{array}$ & Roots & 119 \\
\hline 88 & $\begin{array}{l}\text { 1,6,7-Trihydroxy-3-methyl-4-(1,1,3-trimethyl-2- } \\
\text { buten-1-yl)-9H-xanthen-9-one }\end{array}$ & Root bark & 23 \\
\hline 97 & 16-Hydroxycudratrixanthone Q & Root bark & 145 \\
\hline 98 & 16-Hydroxycudratrixanthone $\mathrm{M}$ & Root bark & 145 \\
\hline 99 & 16-Methoxycudratrixanthone $\mathrm{M}$ & Root bark & 145 \\
\hline
\end{tabular}

\section{Flavonoids}

Flavones

$\begin{array}{ll}100 & \text { Artocarpesin } \\ 101 & \text { Apigenin } \\ 102 & \text { Apigenin-7-O- } \beta \text {-D-glucopyranoside } \\ 103 & \text { Cudraflavone A } \\ 104 & \text { Cudraflavone B } \\ 105 & \text { Cudraflavone C } \\ 106 & \text { Cudraflavone D } \\ 107 & \text { Cudraflavone F } \\ 108 & \text { Cudraflavone G } \\ 109 & \text { Cudraflavone H } \\ 110 & \text { Cycloartocarpesin B } \\ 111 & \text { Cyclomorusin } \\ 112 & \text { Cycloartocarpin } \\ 113 & \text { Hirsutrin/quercetin-3-O- } \beta \text {-D-glucopyranoside } \\ 114 & \text { Kuwanon C } \\ 115 & \text { Licoflavone C } \\ 116 & 5,7,2^{\prime}, 4^{\prime} \text {-Tetrahydroxyflavone/norarthocarpetin } \\ 117 & 6 \text {-Prenylapigenin } \\ 118 & \text { Kaempferol }\end{array}$

$\begin{array}{ll}\text { Roots and stems } & 17 \\ \text { Fruits } & 168 \\ \text { Fruits } & 168 \\ \text { Root bark } & 150 \\ \text { Root bark } & 150 \\ \text { Root bark } & 151 \\ \text { Root bark } & 151 \\ \text { Roots } & 58 \\ \text { Roots } & 58 \\ \text { Root bark } & 145 \\ \text { Roots } & 17 \\ \text { Twigs } & 75 \\ \text { Whole plant } & 152 \\ \text { Fruits } & 66 \\ \text { Roots } & 125 \\ \text { Leaves } & 153 \\ \text { Stems } & 154 \\ \text { Roots } & 58 \\ \text { Root bark } & 134\end{array}$


Table 1 (Contd.)

\begin{tabular}{|c|c|c|c|}
\hline No. & Compound name(s) & Tissue(s) & Ref. \\
\hline 119 & Kaempferol-3-O- $\beta$-D-glucopyranoside/astragalin & Fruits & 168 \\
\hline 120 & Kaempferol-7-O- $\beta$-D-glucopyranoside/populnin & Whole plant & 155 \\
\hline 121 & 6- $p$-Hydroxybenzyl kaempferol-7-O- $\beta$-D-glucopyranoside & Root bark & 156 \\
\hline 122 & Morin & Root bark & 124 \\
\hline 123 & Myricetin & Roots & 117 \\
\hline 124 & Nicotiflorine & Fruits & 168 \\
\hline 125 & Quercetin & Twigs, root bark and stems & 75, 106 and 157 \\
\hline 126 & Quercetin-7-O- $\beta$-D-glucopyranoside/quercimeritrin & Root bark & 158 \\
\hline 127 & 6- $p$-Hydroxybenzyl quercetin-7-O- $\beta$-D-glucopyranoside & Root bark & 156 \\
\hline 128 & Rutin & Fruits & 66 \\
\hline \multicolumn{4}{|c|}{ Flavanones } \\
\hline 129 & Cudraflavanone A & Root bark & 158 \\
\hline 130 & Cudraflavanone B & Roots & 125 \\
\hline 131 & Cudraflavanone C & Roots & 55 \\
\hline 132 & Cudraflavanone D & Roots & 55 \\
\hline 133 & Cudraflavanone E & Roots & 58 \\
\hline 134 & Cudraflavanone $\mathrm{F}$ & Roots & 58 \\
\hline 135 & Cudraflavanone G & Root bark & 145 \\
\hline 136 & (2R)-Cudraflavanone $\mathrm{H}$ & Root bark & 145 \\
\hline 137 & (2S)-Cudraflavanone $\mathrm{H}$ & Root bark & 145 \\
\hline 138 & Cycloaltilisin 7 & Twigs & 75 \\
\hline 139 & Cudracuspiflavanone A & Root bark & 124 \\
\hline 140 & Carthamidin & Leaves & 153 \\
\hline 141 & Dalenin & Root bark & 145 \\
\hline 142 & Dicycloeuchrestaflavanone B & Root bark & 145 \\
\hline 143 & Euchrestaflavanone B & Root bark & 157 \\
\hline 144 & Euchrestaflavanone C & Root bark & 157 \\
\hline 145 & Eriodictyol & Stem bark & 30 \\
\hline 146 & Naringenin & Twigs and root bark & 75 and 157 \\
\hline 147 & Pinocembrin & Root bark & 145 \\
\hline 148 & Prunin & Fruits & 168 \\
\hline 149 & Steppogenin & Twigs, roots and stems & 75,159 and 160 \\
\hline 150 & Tomentosanol D & Root barks & 145 \\
\hline 151 & $\begin{array}{l}(2 S) \text {-2',5,7-Trihydroxy-6-(3-hydroxy-3-methylbutyl)- } \\
\left.6^{\prime \prime}, 6^{\prime \prime} \text {-dimethylpyrano[2 } 2^{\prime \prime}, 3^{\prime \prime}: 4^{\prime}, 5^{\prime}\right] \text { flavanone }\end{array}$ & Roots & 58 \\
\hline 152 & $\begin{array}{l}2^{\prime}, 5,7 \text {-Trihydroxy-4', } 5^{\prime} \text {-(2,2-dimethylchromeno)-8- } \\
\text { (3-hydroxy-3-methylbutyl)flavanone }\end{array}$ & Root bark & 157 \\
\hline 153 & $4^{\prime}$-Hydroxyisolonchocarpin & Root bark & 145 \\
\hline 154 & 5-Dehydroxybavachinone A & Root bark & 145 \\
\hline 155 & $5,7,3^{\prime}, 5^{\prime}$-Tetrahydroxyflavanone & Root bark & 124 \\
\hline 156 & 6-Prenylnaringenin & Roots & 161 \\
\hline 157 & 8-Prenylnaringenin & Root bark & 124 \\
\hline 158 & Aromadendrin/dihydrokaempferol & Root bark and twigs & 75 and 156 \\
\hline 159 & Dihydrokaempferol-7-O- $\beta$-D-glucoside & Twigs & 75 \\
\hline 160 & trans-Dihydromorin & Twigs and whole plant & 133 and 152 \\
\hline 161 & Gericudranin A & Stem bark & 16 \\
\hline 162 & Gericudranin B & Stem bark & 16 \\
\hline 163 & Gericudranin $\mathrm{C}$ & Stem bark & 16 \\
\hline 164 & Gericudranin D & Stem bark & 58 \\
\hline 165 & Gericudranin E & Stem bark & 58 \\
\hline 166 & Taxifolin/dihydroquercetin & Twigs and stems & 75 and 160 \\
\hline 167 & Taxifolin-7-methyl ether & Twigs & 75 \\
\hline 168 & Taxifolin-7-O- $\beta$-D-glucopyranoside & Twigs & 75 \\
\hline 169 & Tricusposide & Bark & 149 \\
\hline 170 & $(2 S, 3 S)$-2,3-trans-Dihydromorin-7-O- $\beta$-D-glucoside & Twigs & 75 \\
\hline 171 & $\begin{array}{l}\text { (2R,3R)-2,3-Dihydro-3,5,6,7-tetrahydroxy-2- } \\
\text { (4-hydroxyphenyl)-4H-1-benzopyran-4-one }\end{array}$ & Root bark & 124 \\
\hline 172 & $3,5,7,2^{\prime}, 4^{\prime}$-Pentahydroxydihydroflavonol & Whole plant & 152 \\
\hline 173 & $5,7,4^{\prime}$-Trihydroxy-8- $p$-hydroxybenzyldihydroflavonol & Root bark & 124 \\
\hline \multicolumn{4}{|c|}{ Isoflavones } \\
\hline 174 & Cudraisoflavone B & Fruits & 28 \\
\hline 175 & Cudraisoflavone C & Fruits & 28 \\
\hline 176 & Cudraisoflavone D & Fruits & 28 \\
\hline
\end{tabular}


Table 1 (Contd.)

\begin{tabular}{|c|c|c|c|}
\hline No. & Compound name(s) & Tissue(s) & Ref. \\
\hline 177 & Cudraisoflavone E & Fruits & 28 \\
\hline 178 & Cudraisoflavone $\mathrm{F}$ & Fruits & 28 \\
\hline 180 & Cudraisoflavone $\mathrm{H}$ & Fruits & 28 \\
\hline 181 & Cudraisoflavone I & Fruits & 28 \\
\hline 182 & Cudraisoflavone J & Fruits & 28 \\
\hline 185 & Cudraisoflavone L (2) & Fruits & 168 \\
\hline 186 & Cudraisoflavone $\mathrm{M}$ & Fruits & 168 \\
\hline 187 & Cudraisoflavone $\mathrm{N}$ & Fruits & 168 \\
\hline 188 & Cudraisoflavone O & Fruits & 168 \\
\hline 189 & Cudraisoflavone $\mathrm{P}$ & Fruits & 168 \\
\hline 190 & Cudraisoflavone Q & Fruits & 168 \\
\hline 195 & Cudracusisoflavone B & Fruits & 66 \\
\hline 196 & Auriculasin & Fruits & 28 \\
\hline 197 & Anagyroidisoflavone A & Fruits & 28 \\
\hline 198 & Alpinumisoflavone & Fruits & 32 \\
\hline 199 & Biochanin A & Root bark & 124 \\
\hline 200 & Erysenegalensein E & Fruits & 31 \\
\hline 201 & Isoerysenegalensein $\mathrm{E}$ & Fruits & 31 \\
\hline 202 & Erythrinin B/wighteone/6-isopentenylgenistein & Twigs and fruits & 28 and 102 \\
\hline 203 & Erythrinin C & Fruits & 28 \\
\hline 204 & Eryvarin B & Fruits & 28 \\
\hline 205 & Erysubin A & Leaves & 87 \\
\hline 206 & Euchrenone b8 & Fruits & 28 \\
\hline 215 & Furowanin B & Leaves & 86 \\
\hline 216 & Gancaonin A & Fruits & 116 \\
\hline 217 & Gancaonin B & Fruits & 28 \\
\hline 218 & Genistein & Twigs and bark & 75 and 149 \\
\hline 219 & Genistein-4'-O- $\beta$-glucopyranoside/sophorobioside & Fruits & 168 \\
\hline 220 & Genistin & Bark & 149 \\
\hline 221 & Glycyrrhisoflavone & Twigs & 75 \\
\hline 222 & Isolupalbigenin & Leaves & 86 \\
\hline 223 & Isochandalone & Fruits & 66 \\
\hline 224 & Lupiwighteone & Fruits & 28 \\
\hline 225 & Lupalbigenin & Leaves & 87 \\
\hline 226 & Laburnetin & Root bark & 145 \\
\hline 227 & Millewanin $\mathrm{H}$ & Leaves & 87 \\
\hline 228 & Millewanin G & Leaves & 153 \\
\hline 229 & Osajin & Fruits & 32 \\
\hline 230 & Orobol & Fruits & 31 \\
\hline 231 & Oroboside & Fruits & 66 \\
\hline 232 & Orobol-8-C-glucoside & Twigs & 75 \\
\hline 233 & Pomiferin & Fruits & 32 \\
\hline 234 & Parvisoflavone A & Root bark & 145 \\
\hline 235 & Senegalensin & Fruits & 31 \\
\hline 236 & Santal & Twigs & 75 \\
\hline 237 & Sphaerobioside & Twigs & 75 \\
\hline 238 & Ulexin B & Fruits & 66 \\
\hline 239 & Ulexone B & Fruits & 66 \\
\hline
\end{tabular}


Table 1 (Contd.)

\begin{tabular}{|c|c|c|c|}
\hline No. & Compound name(s) & Tissue(s) & Ref. \\
\hline 240 & Warangalon & Fruits & 28 \\
\hline 241 & 3'-O-Methylorobol & Root bark & 124 \\
\hline 242 & 4'-O-Methylalpinumisoflavone & Fruits and stem bark & 105 and 116 \\
\hline 243 & 4'-O-Methylcudraisoflavone $\mathrm{O}$ & Fruits & 168 \\
\hline 244 & $4^{\prime}$-O-Methylcudraisoflavone $\mathrm{P}$ & Fruits & 168 \\
\hline 245 & $4^{\prime}$-O-Methylerythrinin C & Fruits & 168 \\
\hline 246 & 4',7-Dihydroxy-5-methoxyisoflavone/5-O-methylgenistein & Stems & 154 \\
\hline 247 & $\begin{array}{l}5,3^{\prime} \text {-Dihydroxy- } 4^{\prime}-\text { methoxy-2 } 2^{\prime \prime}, 2^{\prime \prime}- \\
\text { dimethylpyrano }\left[5^{\prime \prime}, 6^{\prime \prime} ; 6,7\right] \text { isoflavone }\end{array}$ & Fruits & 31 \\
\hline 248 & $\begin{array}{l}5,3^{\prime}, 4^{\prime} \text {-Trihydroxy- } 6^{\prime \prime}, 6^{\prime \prime}- \\
\text { dimethylpyrano }\left[2^{\prime \prime}, 3^{\prime \prime} ; 7,6\right] \text { isoflavone }\end{array}$ & Fruits & 66 \\
\hline 249 & 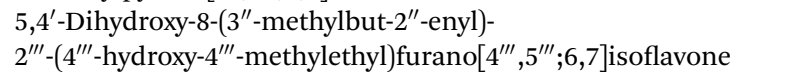 & Fruits & 31 \\
\hline 250 & $\begin{array}{l}5,4^{\prime} \text {-Dihydroxy-6-( }\left(3^{\prime \prime} \text {-methylbut-2 } 2^{\prime \prime} \text {-enyl)- }\right. \\
2^{\prime \prime \prime}-\left(4^{\prime \prime \prime} \text {-hydroxy- } 4^{\prime \prime \prime} \text {-methylethyl)-3"'- }\right. \\
\text { methoxydihydrofurano }\left[4^{\prime \prime \prime}, 5^{\prime \prime \prime} ; 7,8\right] \text { isoflavone }\end{array}$ & Fruits & 31 \\
\hline 251 & $\begin{array}{l}\text { 5,7-Dihydroxy-6-(2"-hydroxy-3"- } \\
\text { methylbut-3"-enyl)-4'-methoxyisoflavone }\end{array}$ & Fruits & 31 \\
\hline 252 & $\begin{array}{l}\text { 5,7, } 4^{\prime} \text {-Trihydroxy-6,8-diprenylisoflavone/ } \\
\text { 6,8-diprenylgenistein } / 8 \text { - }(\gamma, \gamma \text {-dimethylallyl }) \text { wighteone }\end{array}$ & Fruits & 32 and 75 \\
\hline 253 & $5,7,4^{\prime}$-Trihydroxydihydroisoflavone & Whole plant & 152 \\
\hline 254 & 6,8-Diprenylorobol/5,7,3',4'-tetrahydroxy-6,8-diprenylisoflavone & Twigs and fruits & 28 and 75 \\
\hline 255 & 6-Prenylorobol & Leaves & 153 \\
\hline 256 & 7,4'-Dimethoxy-5-hydroxyisoflavone & Fruits & 66 \\
\hline 257 & 8-Hydroxygenistein & Root bark & 145 \\
\hline \multicolumn{4}{|c|}{ Organic acids } \\
\hline 258 & Butyl citrate & Trunk & 15 \\
\hline 259 & Benzoic acid & Fruits & 76 \\
\hline 260 & Boric acid & Fruits & 76 \\
\hline 261 & Citric acid & Fruits & 70 \\
\hline 262 & Mandelic acid & Fruits & 76 \\
\hline 263 & Methyl linoleate & Trunk & 15 \\
\hline 264 & Malic acid & Fruits & 70 \\
\hline 265 & Oxalic acid & Fruits & 70 \\
\hline 266 & Palmitic acid & Trunk & 15 \\
\hline 267 & Palmitic acid methyl ester & Trunk & 15 \\
\hline 268 & Palmitic acid $\beta$-monoglyceride & Trunk & 15 \\
\hline 269 & Protocatechuic acid & Twigs & 75 \\
\hline 270 & Succinic acid & Fruits & 70 \\
\hline 271 & Stearic acid & Trunk & 15 \\
\hline 272 & Syringic acid & Trunk & 15 \\
\hline 273 & Tartaric acid & Fruits & 70 \\
\hline 274 & threo-9,10-O-Isopropylidene-13-hydroxy-(11E)-octadecenoic acid & Roots & 164 \\
\hline 275 & $n$-Decanoic acid & Roots and stems & 10 \\
\hline 276 & $n$-Nonanoic acid & Roots & 10 \\
\hline 277 & $n$-Pentanoic acid & Roots and stems & 10 \\
\hline 278 & $n$-Hexanoic acid & Roots and stems & 10 \\
\hline 279 & $n$-Heptanoic acid & Roots & 10 \\
\hline 280 & $n$-Octanoic acid & Roots & 10 \\
\hline 281 & $(E)$-2-Decenoic acid & Roots & 10 \\
\hline 282 & $(E)$-2-Octenoic acid & Roots and stems & 10 \\
\hline 283 & $2^{\prime}, 3^{\prime}$-Dihydroxypropyl pentadecanoate & Roots & 165 \\
\hline 284 & 4-Hydroxybenzoic acid & Fruits & 168 \\
\hline 285 & 9,12-Octadecadienoic acid & Trunk & 15 \\
\hline 286 & $9,12,15$-Octadecatrienoic acid methyl ester & Trunk & 15 \\
\hline 287 & $9,12,15$-Octadecatrien-1-ol & Trunk & 15 \\
\hline 288 & 9,17-Octadecadienal & Trunk & 15 \\
\hline 289 & $\gamma$-Hexadecalactone & Root bark & 145 \\
\hline \multicolumn{4}{|c|}{ Polysaccharides } \\
\hline 290 & CTP-B1 & Roots & 73 \\
\hline 291 & CTPS-01 & Roots & 72 \\
\hline
\end{tabular}


Table 1 (Contd.)

\begin{tabular}{|c|c|c|c|}
\hline No. & Compound name(s) & Tissue(s) & Ref. \\
\hline 292 & CPS-0 & Roots & 71 \\
\hline 293 & CTPS-1A & Roots & 71 \\
\hline 294 & CTPS-2B & Roots & 71 \\
\hline 295 & CTPS-3A & Roots & 71 \\
\hline \multicolumn{4}{|c|}{ Phenylpropanoids } \\
\hline 296 & Bergapten & Root bark & 145 \\
\hline 297 & Cudrastilbene & Roots & 159 \\
\hline 298 & cis-3', $4^{\prime}$-Diisovalerylkhellactone & Root bark & 145 \\
\hline 299 & Demethylsuberosin & Whole plant & 163 \\
\hline 300 & Decursinol angelate & Root bark & 145 \\
\hline 301 & Gomisin A & Roots & 166 \\
\hline 302 & Gomisin $\mathrm{H}$ & Roots & 166 \\
\hline 303 & Hyuganin C & Root bark & 145 \\
\hline 304 & Imperatorin & Whole plant & 163 \\
\hline 305 & Isoimperatorin & Whole plant & 163 \\
\hline 306 & Oxyresveratrol & Twigs & 75 \\
\hline 307 & Scopoletin & Trunk & 15 \\
\hline 308 & Schizandrin & Whole plant & 166 \\
\hline 309 & Syringaresinol & Whole plant & 166 \\
\hline 310 & Umbelliferone & Root bark & 151 \\
\hline 311 & Xanthyletin & Root bark & 145 \\
\hline 312 & 7-Hydroxy-2H-1-benzopyran-2-one & Trunk & 15 \\
\hline 313 & 5-Methoxy-4,5-diphenyl-2(5H)-furanone & Twigs & 75 \\
\hline 314 & 3-Methyl-2(5H)-furanone & Roots & 10 \\
\hline 315 & 5-Ethyl-2(5H)-furanone & Roots & 10 \\
\hline 316 & 5,5-Dimethyl-2(5H)-furanone & Roots & 10 \\
\hline
\end{tabular}

\section{Other ingredients}

\begin{tabular}{|c|c|}
\hline 317 & Betulin \\
\hline 318 & Butyrospermol \\
\hline 319 & Camphene \\
\hline 320 & Drimenol \\
\hline 321 & Dihydroctinidiolide \\
\hline 322 & Glutinol \\
\hline 323 & Lupeol \\
\hline 324 & Lanosta-8-24-dien-3 $\beta$-ol-acetate \\
\hline 325 & Lanosta-8-en-3-one \\
\hline 326 & Lanosta-7,24-diene-3ß-ol \\
\hline 327 & Lanosta-7,24-diene-3ß-O-acetate \\
\hline 328 & Olean-12-ene \\
\hline 329 & Taraxerone \\
\hline 330 & Terpin hydrate \\
\hline 331 & Ursolic acid \\
\hline 332 & $(E)$-Geranylacetone \\
\hline 333 & $(E)$ - $\beta$-Ionone \\
\hline 334 & (E)-Linalool oxide \\
\hline 335 & (Z)-Linalool oxide \\
\hline 336 & (E)- $\alpha$-Terpineol \\
\hline 337 & $\alpha$-Amyrin \\
\hline 338 & Campesterol \\
\hline 339 & Daucosterol \\
\hline 340 & Itesmol \\
\hline 341 & $\beta$-Sitosterol \\
\hline 342 & $\gamma$-Sitosterol \\
\hline 343 & Achilleol A \\
\hline 344 & Antiarol \\
\hline 345 & Anisaldehyde \\
\hline 346 & Aristolone \\
\hline 347 & Adacene 12 \\
\hline 348 & Brosimine B \\
\hline 349 & Benzophenone \\
\hline 350 & Benzylhydrazine \\
\hline
\end{tabular}

$\begin{array}{ll}\text { Roots } & 165 \\ \text { Fruits } & 168 \\ \text { Roots } & 10 \\ \text { Roots } & 10 \\ \text { Stems } & 10 \\ \text { Root bark } & 145 \\ \text { Roots } & 165 \\ \text { Trunk } & 15 \\ \text { Trunk } & 15 \\ \text { Whole plant } & 152 \\ \text { Whole plant } & 152 \\ \text { Trunk } & 15 \\ \text { Stems } & 160 \\ \text { Roots } & 10 \\ \text { Roots } & 165 \\ \text { Roots } & 10 \\ \text { Roots and stems } & 10 \\ \text { Roots } & 10 \\ \text { Roots } & 10 \\ \text { Roots and stems } & 10 \\ \text { Root bark } & 145 \\ \text { Trunk } & 15 \\ \text { Roots } & 165 \\ \text { Roots } & 165 \\ \text { Roots } & 165 \\ \text { Trunk } & 15 \\ \text { Whole plant } & 163 \\ \text { Fruits } & 76 \\ \text { Roots } & 10 \\ \text { Trunk } & 15 \\ \text { Fruits } & 76 \\ \text { Root bark } & 145 \\ \text { Stems } & 10 \\ \text { Fruits } & 76 \\ & \end{array}$


Table 1 (Contd.)

\begin{tabular}{|c|c|c|c|}
\hline No. & Compound name(s) & Tissue(s) & Ref. \\
\hline 351 & Bis(2-azabicyclo[2.2.1]hept-5-en-2-yl)diazene & Fruits & 76 \\
\hline 352 & Butylated hydroxytoluene & Fruits & 76 \\
\hline 353 & Cudracuspiphenone A & Roots & 116 \\
\hline 354 & Cudracuspiphenone B & Roots & 116 \\
\hline 355 & Cudrachromone A & Root bark & 145 \\
\hline 356 & Cudraphenol A & Root bark & 145 \\
\hline 357 & Cudraphenol B & Root bark & 145 \\
\hline 358 & Cudraphenol C & Root bark & 145 \\
\hline 359 & Cudraphenone E & Root bark & 145 \\
\hline 360 & Cudradihydrochalcone A & Fruits & 168 \\
\hline 361 & Cudrabibenzyl A & Fruits & 168 \\
\hline 362 & $(E)$-Cinnamic aldehyde & Roots and stems & 10 \\
\hline 363 & Dopamine & Fruits & 76 \\
\hline 364 & Demeton-O-methyl & Fruits & 76 \\
\hline 365 & Diethyl phthalate & Fruits & 76 \\
\hline 366 & Ethyl- $N$-methylcarbamate & Fruits & 76 \\
\hline 367 & Eriosematin A & Root bark & 145 \\
\hline 368 & Ethyl $p$-tert-butylbenzoic acid & Fruits & 76 \\
\hline 369 & Eugenol & Roots and stems & 10 \\
\hline 370 & Isoeugenol & Roots & 10 \\
\hline 371 & Isoencecalin & Root bark & 145 \\
\hline 372 & Indene & Fruits & 76 \\
\hline 373 & Lavender lactone & Roots & 10 \\
\hline 374 & Lycopene & Fruits & 77 \\
\hline 375 & Lutein & Fruits & 77 \\
\hline 376 & Palustrol & Fruits & 76 \\
\hline 377 & Peonoside & Bark & 149 \\
\hline 378 & Phenol & Fruits, roots and stems & 10 and 76 \\
\hline 379 & Phytofluene & Fruits & 77 \\
\hline 380 & Phenylethyl alcohol & Roots and stems & 10 \\
\hline 381 & Pyridine & Roots & 10 \\
\hline 382 & Pyrrole-2-carboxaldehyde & Stems & 10 \\
\hline 383 & Ruboxanthin & Fruits & 77 \\
\hline 384 & Salicylamide & Fruits & 76 \\
\hline 385 & Scyllitol & Fruits & 76 \\
\hline 386 & Sucrose & Bark & 149 \\
\hline 387 & Stachydrine & Roots & 167 \\
\hline 388 & Tridecanol & Fruits & 76 \\
\hline 389 & Undecane & Fruits & 76 \\
\hline 390 & Vanillin & Root bark & 145 \\
\hline 391 & Zeaxanthin & Fruits & 77 \\
\hline 392 & $p$-Vinylguaiacol & Roots and stems & 10 \\
\hline 393 & $n$-Hexanal & Roots & 10 \\
\hline 394 & $n$-Hexanol & Roots & 10 \\
\hline 395 & $n$-Butanol & Roots & 10 \\
\hline 396 & $n$-Nonanal & Roots and stems & 10 \\
\hline 397 & $N$-Acetylnorephedrine & Fruits & 76 \\
\hline 398 & 1-Phenyl-1-cyclohexylethane & Fruits & 76 \\
\hline 399 & 1-Methyl-2-pyrrolidone & Roots and stems & 10 \\
\hline 400 & 1-[(2,4,6-Trimethylphenyl)methyl]imidazole & Fruits & 76 \\
\hline 401 & $(4 S)$-1,1-Difluoro-4 vinylspiropentane & Fruits & 76 \\
\hline 402 & 2-Deuteriophenylalanine & Fruits & 76 \\
\hline 403 & 2-Furanmethanol & Trunk & 15 \\
\hline 404 & 2-Ethyl-1-hexanol & Roots and stems & 10 \\
\hline 405 & 2-Acetylpyrrole & Stems & 10 \\
\hline 406 & 2,3-Dihydro-3,5-dihydroxy-6-methyl-4H-pyran-4-one & Trunk & 15 \\
\hline 407 & 2,4-Bis(4-hydroxybenzyl)phenol & Root bark and stems & 10 and 145 \\
\hline 408 & 2,5-Furandione & Trunk & 15 \\
\hline 409 & 3-Methoxycarbonylindole & Root bark & 145 \\
\hline 410 & 3-Methyl-2,5-furandione & Trunk & 15 \\
\hline 411 & 4-Acetylpyrazole & Roots & 10 \\
\hline 412 & 4-Ethylguaiacol & Roots and stems & 10 \\
\hline 413 & 4-Hydroxybenzalacetone & Root bark & 145 \\
\hline
\end{tabular}


Table 1 (Contd.)

\begin{tabular}{|c|c|c|c|}
\hline No. & Compound name(s) & Tissue(s) & Ref. \\
\hline 414 & 4-Hydroxymethylbenzoate & Root bark & 145 \\
\hline 415 & 4-(Methoxymethyl)phenol & Whole plant & 163 \\
\hline 416 & 4-Methyltridecane & Fruits & 76 \\
\hline 417 & 4-Methylguaiacol & Roots & 10 \\
\hline 418 & 4-Hydroxybenzaldehyde/ $p$-hydroxybenzaldehyde & Trunk & 15 \\
\hline 429 & 4 -Valerolactone & Roots and stems & 10 \\
\hline 420 & 4,4-Diphenyl-5-methyl-2-cyclohexenone & Fruits & 76 \\
\hline 421 & $p$-Hydroxybenzyl alcohol & Trunk & 15 \\
\hline 422 & 5-(Hydroxymethyl)-2-furancarboxaldehyde & Trunk & 15 \\
\hline 423 & 5-Hydroxy-2,2-dimethyl-2H,6H-benzodipyran-6-one & Root bark & 124 \\
\hline 424 & 5-Methyl-1H-pyrrole-2-carboxaldehyde & Stems & 10 \\
\hline 425 & 5,7-Dihydroxychromone & Root bark & 124 \\
\hline 426 & 6-Pentyl-5,6-dihydro- $2 H$-pyran-2-one & Roots and stems & 10 \\
\hline 427 & 8-Chloro-6-(2-fluorophenyl)imidazole[1,2- $a][1,4]$ benzodiazepine & Fruits & 76 \\
\hline 428 & $\alpha$-Carotene & Fruits & 77 \\
\hline 429 & $\beta$-Carotene & Fruits & 77 \\
\hline 430 & Neo- $\beta$-carotene & Fruits & 77 \\
\hline 431 & $\gamma$-Amylbutyrolactone & Roots and stems & 10 \\
\hline 432 & $\gamma$-Butylbutyrolactone & Roots and stems & 10 \\
\hline 433 & $\gamma$-Butyrolactone & Roots & 10 \\
\hline 434 & $\gamma$-Caprolactone & Roots & 10 \\
\hline 435 & $\gamma$-Crotonolactone & Roots & 10 \\
\hline 436 & $\gamma$-Dodecalactone & Roots and stems & 10 \\
\hline 437 & $\gamma$-Palmitolactone & Roots & 10 \\
\hline 438 & Arginine & Roots & 167 \\
\hline 439 & Alanine & Roots & 167 \\
\hline 440 & Aspartate & Roots & 167 \\
\hline 441 & Glutamic acid & Roots & 167 \\
\hline 442 & Proline & Roots & 167 \\
\hline 443 & Polycopene & Fruits & 77 \\
\hline
\end{tabular}

immunomodulatory activities, namely, CTP-B1 (290), CTPS-01 (291), CPS-0 (292), CTPS-1A (293), CTPS-2B (294) and CTPS-3A (295), were obtained from the roots of C. tricuspidata. $^{72-74}$ Their backbones were revealed to be commonly substituted with $\alpha$-D-glucuronic acid, 4-O-methyl- $\alpha$-D-glucuronic acid, and neutral sugar units such as $\alpha$-L-arabinose, $\alpha$-D-xylose and $\alpha$-Dgalactose. $^{74}$

\subsection{Phenylpropanoids (296-316)}

Twenty-one phenylpropanoids have been reported in C. tricuspidata, such as oxyresveratrol (306), ${ }^{75}$ scopoletin (307), ${ }^{15} 3$ methyl-2(5H)-furanone $(\mathbf{3 1 4}),{ }^{10}$ and $\quad 5$-ethyl-2 $(5 H)$-furanone (315). ${ }^{10}$ Oxyresveratrol, as a representative phenylpropanoid, was isolated from the twigs of $C$. tricuspidata, exhibited potent inhibitory activity against mushroom tyrosinase and might serve as an anti-browning agent for food..$^{75}$

\subsection{Others $(317-443)$}

In addition to the aforementioned components, a large number of other components have also been identified in C. tricuspidata. Twenty-nine compounds were identified in the essential oil of C. tricuspidata fruits, which accounted for $94.46 \%$ of the essential oil, such as demeton- $O$-methyl (364), diethyl phthalate (365), ethyl- $N$-methylcarbamate (366), indene (372), scyllitol
(385), tridecanol (388) and 1-phenyl-1-cyclohexylethane (398). ${ }^{76}$ It should be mentioned that a series of carotenoids were identified in C. tricuspidata fruits, including lycopene (374), lutein (375), phytofluene (379), ruboxanthin (383), zeaxanthin (391), $\alpha$ carotene (428), $\beta$-carotene (429), neo- $\beta$-carotene (430) and polycopene (443). ${ }^{77}$

\section{Pharmacological properties}

Accumulated studies have revealed that the extracts and components of $C$. tricuspidata exhibited a broad spectrum of pharmacological activities, including anti-inflammatory, ${ }^{22,23}$ antioxidant, ${ }^{78,79}$ antitumor, ${ }^{16,24}$ hepatoprotective, ${ }^{25,26}$ neuroprotective, ${ }^{, 7,28}$ antiobesity, ${ }^{18,19}$ immunomodulatory, ${ }^{70,71}$ antiatherosclerotic, ${ }^{80,81}$ antimicrobial, ${ }^{11,76}$ skin-protecting, ${ }^{79,82}$ and antidiabetic ${ }^{21,52}$ effects. The presence of a variety of bioactive compounds may be synergistically or individually responsible for the various activities of this species (Fig. 3). Among these, xanthones and flavonoids are representative ingredients that mainly possess anti-inflammatory, antioxidant and antitumor activities.

\subsection{Anti-inflammatory activity}

There has been strong evidence that diseases associated with inflammation may be ameliorated by C. tricuspidata. The anti- 


\section{Xanthones}
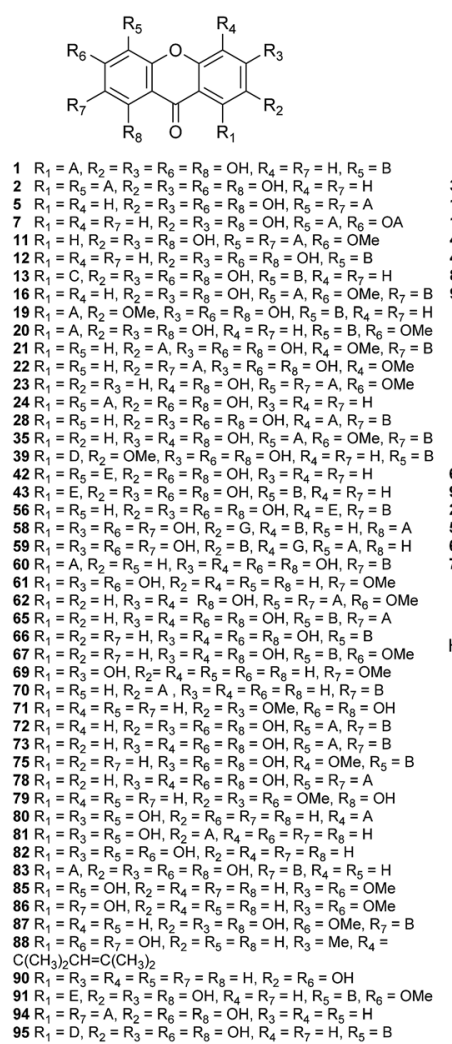

$\begin{array}{ll}1 & R_{1}=A, R_{2}=R_{3}=R_{6}=R_{8}=O H, R_{4}=R_{7}=H, R_{5}=B \\ 2 & R_{1}=R_{5}=A_{1}, R_{2}=R_{3}=R_{6}=R_{8}=O H, R_{4}=R_{7}=H\end{array}$

$\begin{array}{ll}5 & R_{1}=R_{4}=H, R_{2}=R_{3}=R_{6}=R_{8}=O H, R_{5}=R_{7}=A \\ 7 & R_{1}=R_{4}=R_{7}=R_{7}=R_{2}=R_{3}=R_{8}=O H, R_{8}=A, R_{6}=O A\end{array}$

$11 R_{1}=R_{4}=R_{7}=R_{3}, R_{2}=R_{3}=R_{1}$

$12 R_{1}=R_{4}=R_{7}=H, R_{2}=R_{3}=R_{6}=R_{8}=O H, R_{5}=B$

$16 R_{1}=R_{4}=H, R_{2}=R_{3}=R_{8}=O H, R_{5}=A, R_{6}=O M R_{1}, R_{7}=$

$20 R_{1}=A, R_{2}=R_{3}=R_{8}=O H, R_{4}=R_{7}=H_{3} R_{5}=B_{4} R_{6}=O M e$

$22 R_{1}=R_{5}=H, R_{2}=A_{1}, R_{3}=R_{6}=R_{8}=O H, R_{4}=O M R_{2}, R_{7}=B$

$23 R_{1}=R_{2}=R_{3}=H, R_{4}=R_{8}=O H, R_{5}=R_{7}=A, R_{6}=O M$

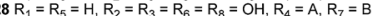

$35 R_{1}=R_{2}=H, R_{3}=R_{4}=R_{8}=O H_{1}, R_{5}=A_{A}, R_{6}=O M e, R_{7}=B$
$39 R_{1}=D, R_{2}=O M e, R_{3}=R_{6}=R_{8}=O H, R_{4}=R_{7}=H, R_{5}=B$

$42 R_{1}=R_{5}=E, R_{2}=R_{6}=R_{8}=O H, R_{3}=R_{4}=R_{7}=H$

$56 R_{1}=R_{5}=H, R_{2}=R_{3}=R_{6}=R_{8}=O H, R_{4}=E, R_{7}=B$

$59 R_{1}=R_{3}=R_{6}=R_{7}=O H, R_{2}=B, R_{4}=G, R_{5}=A, R_{8}=H$

$60 R_{1}=A_{1}, R_{2}=R_{5}=H, R_{3}=R_{4}=R_{6}=R_{8}=O H, R_{7}=B$
$61 R_{1}=R_{3}=R_{6}=O H R_{2}=R_{4}=R_{5}=R_{6}=H, R_{7}=O M$

$62 R_{1}=R_{2}=H, R_{3}=R_{4}=R_{8}=O H, R_{5}=R_{7}=A, R_{6}=O$

$B, R_{6}=O N$

列 $=R_{3}=H_{1}, R_{7}=B$

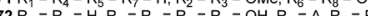

$73 R_{1}=R_{2}=H, R_{3}=R_{4}=R_{6}=R_{8}=O H, R_{5}=A, R_{7}=B$

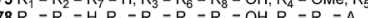

$79 R_{1}=R_{4}=R_{5}=R_{7}=H, R_{2}=R_{3}=R_{8}=O M e R_{8}=O H$

$80 R_{1}=R_{3}=R_{5}=O H_{1}, R_{2}=R_{6}=R_{7}=R_{8}=H_{1}, R_{4}=A$
$81 R_{1}=R_{3}=R_{5}=O H, R_{2}=A, R_{4}=R_{6}=R_{7}=R_{8}=H$

$83 R_{1}=A, R_{2}=R_{3}=R_{8}=R_{5}=O R_{2} R_{7}=B, R_{1}=R_{5}=$

$87 R_{1}=R_{4}=R_{5}=H, R_{2}=R_{3}=R_{8}=O H, R_{6}=O M e, R_{7}=B$

$\mathrm{C}\left(\mathrm{CH}_{3}\right)_{2} \mathrm{CH}=\mathrm{C}\left(\mathrm{CH}_{3}\right.$

$91 R_{1}=E_{E} R_{2}=R_{3}=R_{8}=O H, R_{4}=R_{7}=H, R_{5}=B, R_{6}=O M$

${ }_{95} R_{1}=D R_{2}=R_{3}=R_{6}=R_{8}=O H R_{4}=R_{7}=H_{3} R_{5}=B$
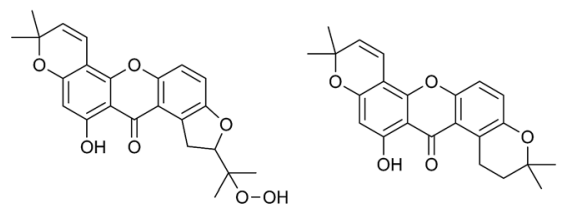

39
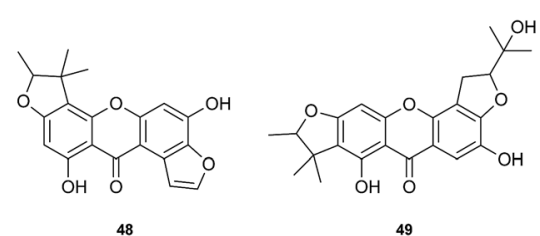

$18 R_{1}=H, R_{2}=B$
$27 R_{1}=B, R_{2}=H$
$76 R_{1}=R_{2}=H$

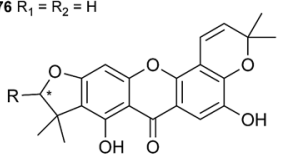

$32(14 S) \mathrm{R}=\mathrm{Me}$
$46 \mathrm{R}=\mathrm{CH}_{2} \mathrm{OH}$

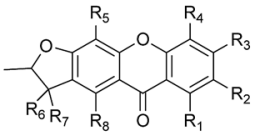<smiles>[R7]c1cc2oc3c(O)cc4c(c3c(=O)c2c(O)c1O)C=CC(C)(C)O4</smiles>

40
$8 R_{1}=R_{3}=R_{4}=O H, R_{2}=B, R_{5}=R_{6}=H$

$6 R_{1}=R_{4}=H, R_{2}=R_{3}=R_{8}=O H, R_{5}=A, R_{6}=R_{7}=M e$

$9 R_{1}=R_{4}=R_{5}=H, R_{2}=R_{3}=R_{8}=O H, R_{6}=R_{7}=M e$
$29 R_{1}=R_{5}=H, R_{2}=R_{3}=R_{8}=O H, R_{4}=A, R_{6}=R_{7}=M e$

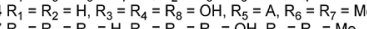<smiles>CC(C)=CCc1c(O)cc(O)c2c(=O)c3c4c(ccc3oc12)CC(C(C)(C)C)O4</smiles>

26

30

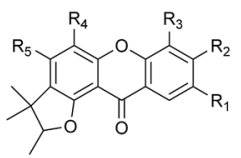

$33 R_{1}=H, R_{2}=R_{3}=O H, R_{4}=A, R_{5}=O M e$ $34 R_{1}=R_{2}=O H, R_{3}=H, R_{4}=A, R_{5}=O M e$
$44 R_{1}=R_{2}=R_{5}=O H, R_{3}=A, R_{4}=H$
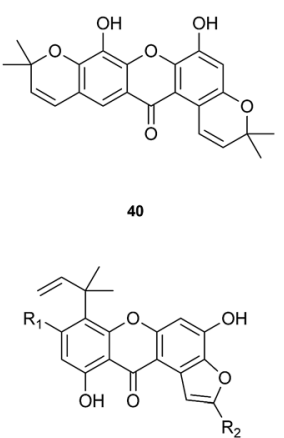

$51 \mathrm{R}_{1}=\mathrm{OH}, \mathrm{R}_{2}=\mathrm{C}=\mathrm{CH}_{2} \mathrm{CH}_{3}$
$54 \mathrm{R}_{1}=\mathrm{OMe}, \mathrm{R}_{2}=\mathrm{C}\left(\mathrm{CH}_{3}\right)_{2} \mathrm{OH}$<smiles></smiles><smiles>CC1Oc2cc3oc4c(O)c5c(cc4c(=O)c3c(O)c2C1(C)C)C=CC(C)(C)O5</smiles>

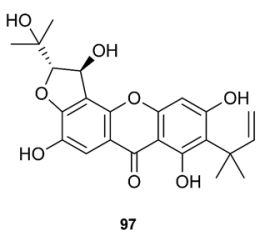

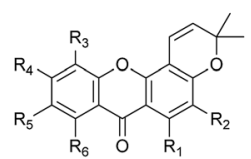

$4 R_{1}=R_{4}=R_{5}=O H, R_{2}=A, R_{3}=R_{6}=H$

$14 R_{1}=R_{4}=R_{5}=O H, R_{2}=B, R_{3}=R_{6}=H$

$25 R_{1}=R_{5}=O H, R_{2}=R_{3}=R_{4}=H, R_{6}=A$
$38 R_{1}=R_{5}=O H, R_{2}=R_{3}=R_{4}=H, R_{6}=E$

$38 R_{1}=R_{5}=O H_{1} R_{2}=R_{3}=R_{4}=H, R_{6}=E$
$68 R_{1}=R_{3}=H, R_{2}=R_{4}=R_{6}=O H, R_{5}=B$

$68 R_{1}=R_{3}=H, R_{2}=R_{4}=R_{6}=O H, R_{5}=$
$74 R_{1}=R_{5}=O H, R_{2}=R_{3}=R_{4}=R_{6}=H$

$84 R_{1}=R_{5}=H_{1}, R_{2}=R_{4}=R_{6}=O H H_{6}, R_{3}=B$
$93 R_{1}=R_{3}=O H, R_{2}=R_{4}=R_{5}=R_{6}=H$
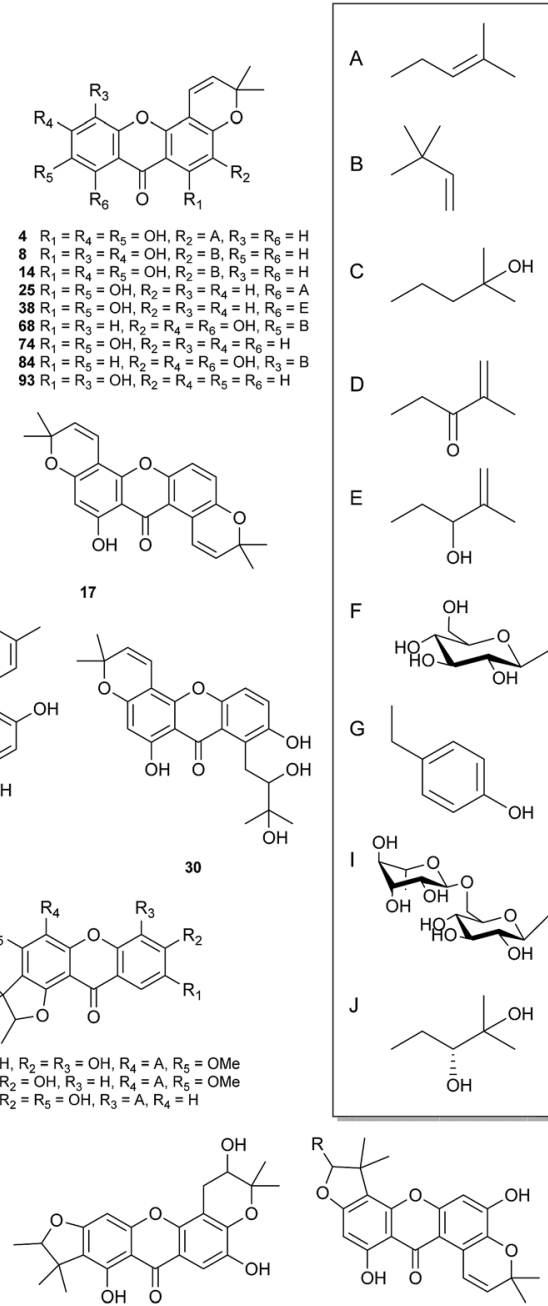

$47 \mathrm{R}=\mathrm{CH}_{2} \mathrm{OH}$<smiles>C=CC(C)(C)c1c(O)cc(O)c2oc3cc(O)c4c(c3c(=O)c12)CC(C(C)(C)O)O4</smiles>

52

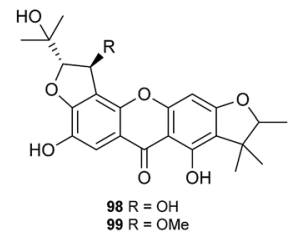

Fig. 2 Chemical structures of xanthones and flavonoids isolated from C. tricuspidata.

inflammatory molecular mechanisms could be elucidated on the basis of the effects of the extracts and compounds from $C$. tricuspidata (Fig. 4). It has been reported that a methanolic extract of $C$. tricuspidata could decrease the production of the pro-inflammatory cytokines interleukin-2 (IL-2) and interferon$\gamma($ IFN- $\gamma$ ) by selectively inhibiting the proliferation of anti-CD3/ CD28-mediated $\mathrm{CD}^{+} \mathrm{CD} 25^{-}$T-cells. ${ }^{15}$ The chloroform $\left(\mathrm{CHCl}_{3}\right)$ fraction of $C$. tricuspidata was observed to inhibit the overproduction of nitric oxide (NO) and prostaglandin $\mathrm{E}_{2}\left(\mathrm{PGE}_{2}\right)$ by decreasing the expression of inducible nitric oxide synthase (iNOS) and cyclooxygenase-2 (COX-2) and reducing the levels of tumor necrosis factor- $\alpha$ (TNF- $\alpha$ ), IL-1 $\beta$ and IL-6 in RAW 264.7 mouse macrophage cells stimulated with lipopolysaccharide (LPS). ${ }^{83}$ The ethyl acetate (EtOAc) fraction of the stem bark could 
Flavonoids

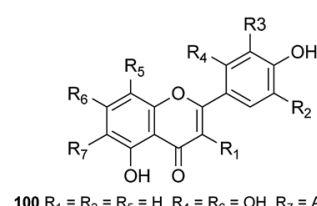

$100 R_{1}=R_{2}=R_{5}=H, R_{4}=R_{6}=O H, R_{7}=A$ $101 R_{1}=R_{2}=R_{3}=R_{4}=R_{5}=R_{7}=H, R_{6}=O H$ $102 R_{1}=R_{2}=R_{3}=R_{4}=R_{5}=R_{7}=H, R_{6}=O F$ $105 R_{1}=R_{7}=A, R_{2}=R_{5}=H, R_{4}=R_{6}=O H$ $106 R_{1}=R_{5}=H, R_{2}=R_{7}=A, R_{4}=R_{6}=O H$ $113 R_{1}=A, R_{2}=R_{5}=H, R_{4}=R_{6}=O H, R_{7}=R_{2}=R_{4}=R_{2}-E$
$R_{7}=R_{7}=H, R_{3}=R_{6}=O H$ $114 R_{1}=R_{5}=A, R_{2}=R_{7}=H, R_{4}=R_{6}=O H$ $115 R_{1}=R_{2}=R_{4}=R_{7}=H, R_{5}=A, R_{6}=O H$ $116 R_{1}=R_{2}=R_{5}=R_{7}=H, R_{4}=R_{6}=O H$ $117 R_{1}=R_{2}=R_{4}=R_{5}=H, R_{6}=O H, R_{7}=A$ 18 $R_{1}=R_{6}=O H, R_{2}=R_{3}=R_{4}=R_{5}=R_{7}=H$ $119 \mathrm{R}_{1}=\mathrm{OF}, \mathrm{R}_{2}=\mathrm{R}_{3}=\mathrm{R}_{4}=\mathrm{R}_{5}=\mathrm{R}_{7}=\mathrm{H}, \mathrm{R}_{6}=\mathrm{OH}$ $121 R_{1}=O H, R_{2}=R_{3}=R_{4}=H, R_{6}=O F, R_{7}=G$ $122 R_{1}=R_{6}=R_{4}=O H, R_{2}=R_{3}=R_{5}=R_{7}=H$ $123 R_{1}=R_{2}=R_{3}=R_{6}=O H, R_{4}=R_{5}=R_{7}=H$ $124 R_{1}=O \mathrm{Ol}_{2}=R_{2}=R_{4}=R_{5}=R_{7}=H, R_{6}=O H$ $125 R_{1}=R_{3}=R_{6}=O H, R_{2}=R_{4}=R_{5}=R_{7}=H$ $126 R_{1}=R_{3}=O H, R_{2}=R_{4}=R_{5}=R_{7}=H, R_{6}=O F$ $128 R_{1}=O I_{2}, R_{2}=R_{4}=R_{5}=R_{7}=H, R_{6}=R_{6}=R_{7}$<smiles>[R]c1cc([C@H]2CC(=O)c3c(O)c([R])c([R])c([R])c3O2)c([R])c([R])c1[R]</smiles><smiles>CC(C)=CC1Oc2cc(O)ccc2-c2oc3c4c(cc(O)c3c(=O)c21)OC(C)(C)C=C4</smiles>

111<smiles>[R]c1cc(O)c([R])c([R])c1-c1oc2cc3c(c(O)c2c(=O)c1[R])C=CC(C)(C)O3</smiles>
$104 R_{1}=A, R_{2}=R_{3}=H, R_{4}=O H$
$108 R_{1}=R_{3}=A, R_{2}=O H, R_{4}=H$
$110 R_{1}=R_{2}=R_{3}=H, R_{4}=O H$<smiles>COc1cc2oc3c(c(=O)c2c(O)c1/C=C/C(C)C)C(C=C(C)C)Oc1cc(O)ccc1-3</smiles>

112

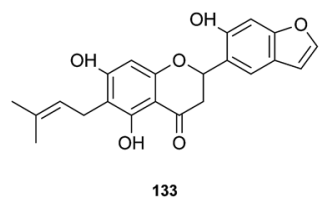<smiles>CC(C)=CCc1c(O)cc2c(c1O)C(=O)CC(c1cc3c(cc1O)OC(C)(C)C(O)C3)O2</smiles>

134<smiles>[R]c1cc2c(cc1-c1cc(=O)c3c(O)c([R])c([R])c([R])c3o1)C=CC(C)(C)O2</smiles>

$129(2 S) R_{1}=A, R_{2}=R_{4}=O H, R_{3}=H$ 135 (2S) $R_{1}=E, R_{2}=R_{4}=O H, R_{3}=H$ $144(2 S) R_{1}=H, R_{2}=R_{4}=O H, R_{3}=A$ $151(2 S) R_{1}=R_{4}=H, R_{2}=O H, R_{3}=\mathrm{C}$
$152(2 R) R_{1}=A, R_{2}=R_{4}=O H, R_{3}=H$
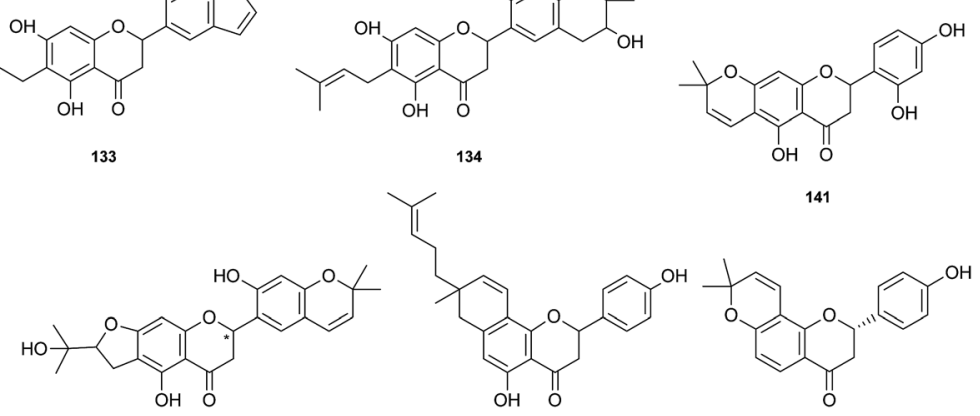

$30 R_{1}=R_{4}=R_{6}=O H, R_{2}=A, R_{3}=R_{5}=R_{7}=H$ $131 R_{1}=R_{6}=O H, R_{2}=R_{5}=A, R_{3}=R_{5}=R_{6}=R_{7}$ $132 R_{1}=R_{4}=R_{6}=O H, R_{2}=R_{3}=A, R_{5}=R_{7}=H$
$139 R_{4}=R_{4}=R_{1}$ $140 R_{1}=R_{2}=R_{4}=O H, R_{3}=R_{5}=R_{5}=R_{7}=O H$ $143 R_{1}=R_{4}=R_{6}=O H, R_{2}=R_{3}=A, R_{5}=R_{7}=H$ $145 R_{1}=R_{4}=R_{5}=O H, R_{2}=R_{3}=R_{6}=R_{7}=H$ $146 R_{1}=R_{4}=O H, R_{2}=R_{3}=R_{5}=R_{6}=R_{7}=H$ $147 R_{1}=O H, R_{2}=R_{3}=R_{4}=R_{5}=R_{6}=R_{7}=H$ $148 R_{1}=O F_{1} R_{2}=R_{3}=R_{5}=R_{6}=R_{7}=H, R_{4}=O H$

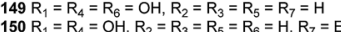
$155 \mathrm{R}_{1}=\mathrm{R}_{4}=\mathrm{R}_{5}=\mathrm{OH}, \mathrm{R}_{2}=\mathrm{R}_{4}=\mathrm{R}_{5}=\mathrm{R}_{7} \mathrm{R}_{7}=\mathrm{H}$ $156 R_{1}=R_{4}=O H, R_{2}=A, R_{3}=R_{5}=R_{6}=R_{7}=H$ $157 R_{1}=R_{4}=O H, R_{2}=R_{3}=R_{5}=R_{6}=H, R_{7}=A$<smiles>[R5]c1c(O)ccc([C@H]2Oc3c([R])c([R])c([R])c(O)c3C(=O)[C@H]2O)c1[R]</smiles>

$158 R_{1}=R_{3}=R_{4}=R_{5}=H, R_{2}=O H$ $158 R_{1}=R_{3}=R_{4}=R_{5}=H, R_{2}=O H$
$159 R_{1}=R_{3}=R_{4}=R_{5}=H, R_{2}=O F$ $160 R_{1}=R_{3}=R_{5}=H_{1}, R_{2}=R_{4}=O H$
$161 R_{1}=R_{3}=G_{3} R_{2}=R_{5}=O H, R_{4}=H$ $161 R_{1}=R_{3}=G, R_{2}=R_{5}=O H, R_{4}=H$
$162 R_{1}=R_{4}=H, R_{2}=R_{5}=O H, R_{3}=G$ $163 R_{1}=G, R_{2}=R_{5}=O H, R_{3}=R_{4}=$ $164 R_{1}=R_{3}=G, R_{2}=O H, R_{4}=R_{5}=$ $165 R_{1}=R_{1}, R_{2}=R_{2}=R_{3}=R_{4}=R_{5}=H$
$166 R_{1}=R_{3}=R_{4}=H, R_{2}=R_{5}=O H$ $167 R_{1}=R_{3}=R_{4}=H, R_{2}=O M R_{5}=O H$ $168 R_{1}=R_{3}=R_{4}=H_{1} R_{2}=O F, R_{5}=O H$ $169 R_{1}=G, R_{2}=O F, R_{3}=R_{4}=H, R_{5}=O H$ $171 R_{1}=R_{2}=O H, R_{3}=R_{4}=R_{5}=H$ $172 R_{1}=R_{4}=R_{5}=H, R_{2}=R_{3}=O H$
$173 R_{1}=R_{4}=R_{5}=H, R_{2}=O H, R_{3}=G$<smiles>CC1(C)C=Cc2cc([C@H]3CC(=O)c4c(cc5c(c4O)C=CC(C)(C)O5)O3)c(O)cc2O1</smiles>

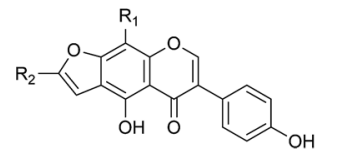

$174 \mathrm{R}_{1}=\mathrm{A}, \mathrm{R}_{2}=\mathrm{H}$ $205 \mathrm{R}_{1}=\mathrm{H}, \mathrm{R}_{2}=\mathrm{C}\left(\mathrm{CH}_{3}\right)_{2} \mathrm{OH}$ $249 \mathrm{R}_{1}=\mathrm{A}, \mathrm{R}_{2}=\mathrm{C}\left(\mathrm{CH}_{3}\right)_{2} \mathrm{OH}$<smiles>Cc1ccc([C@H]2CC(=O)c3c(cc4c(c3O)CC(C(C)(C)C)O4)O2)cc1</smiles>

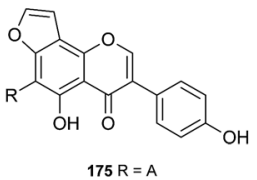

$175 R=A$
$176 R=D$
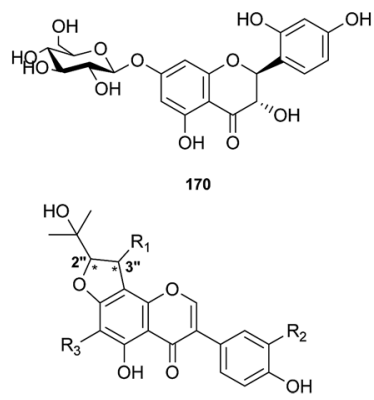

$177 R_{1}=R_{2}=H, R_{3}=E$ $215 \mathrm{R}_{1}=\mathrm{H}, \mathrm{R}_{2}=\mathrm{OH}, \mathrm{R}_{3}=\mathrm{A}$ $235 R_{1}=R_{2}=H, R_{3}=A$
$250\left(2^{\prime \prime} R, 3^{\prime \prime} R\right) R_{1}=O M e, R_{2}=H, R_{3}=A$

Fig. 2 (contd.)

suppress the production of NO and expression of iNOS in RAW 264.7 cells stimulated with IFN- $\gamma /$ LPS via the inactivation of nuclear factor- $\mathrm{KB}$ (NF-kB). ${ }^{84}$ The EtOAc fraction of C. tricuspidata stem bark could inhibit the differentiation of osteoclasts stimulated by IL-1 $\beta$ and mediated by receptor activator of NF-KB ligand, the phosphorylation of extracellular signal-regulated kinase (ERK) 1/2 and p38 mitogen-activated protein kinase (MAPK), and the expression of c-Fos and nuclear factor of activated T-cells c1 (NFATc1).$^{85}$ The EtOAc fraction of the whole plant was also found to reduce the expression of IL-1 $\beta$, matrix metalloproteinases (MMPs), COX-2 and $\mathrm{PGE}_{2}$ by inhibiting the phosphorylation of MAPK and the activation of NF- $\mathrm{KB}$ signalling pathways in rheumatoid synovial fibroblasts. ${ }^{86}$ The above research suggested that $C$. tricuspidata may be useful for managing bone destruction in inflammatory diseases, such as rheumatoid arthritis (RA).

Numerous compounds from C. tricuspidata possess noticeable anti-inflammatory properties. Prenylated isoflavones from the leaves of C. tricuspidata, including cudraisoflavone L (184), wighteone (202) and furowanin B (215) exhibited potential antiinflammatory activity by inhibiting the production of NO in LPS-stimulated RAW 264.7 cells, with inhibition values of 72.5 $\pm 2.4 \%, 66.9 \pm 1.8 \%$, and $55.4 \pm 2.7 \%$ at a concentration of 10 $\mu \mathrm{M}$, respectively. ${ }^{87}$ It was found that the position of hydroxyl groups in the xanthone moiety was important for the NOinhibiting activity, and the catechol moiety was partially responsible for the inhibitory activity (Table 2).$^{88} \mathrm{~A} \mathrm{C}$. tricuspidata glycoprotein suppressed the expression of iNOS and COX-2 

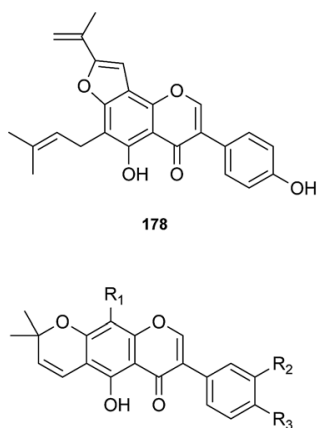

$179 \mathrm{R}_{1}=\mathrm{G}, \mathrm{R}_{2}=\mathrm{H}, \mathrm{R}_{3}=\mathrm{OH}$
$186 \mathrm{R}_{1}=\mathrm{H}, \mathrm{R}_{2}=\mathrm{J}, \mathrm{R}_{3}=\mathrm{OH}$ $186 R_{1}=H, R_{2}=J, R_{3}=O H$
$196 R_{1}=A, R_{2}=R_{3}=O H$ $198 R_{1}=R_{2}=H, R_{3}=O H$ $206 \mathrm{R}_{1}=\mathrm{D}, \mathrm{R}_{2}=\mathrm{H}, \mathrm{R}_{3}=\mathrm{OH}$ $240 R_{1}=A_{1} R_{2}=H, R_{3}=O H$
$242 R_{1}=R_{2}=H_{1} R_{3}=O M e$ $242 R_{1}=R_{2}=H_{1} R_{3}=O M e$ $247 R_{1}=H_{1}, R_{2}=O H, R_{3}=O M C$
$248 R_{1}=H, R_{2}=R_{3}=O H$<smiles>[R6]c1cc(-c2coc3c([R3])c([R6])c([R3])c([R])c3c2=O)cc([R2])c1[R]</smiles>

$184 R_{1}=A, R_{2}=R_{4}=R_{5}=R_{7}=O H, R_{3}=C_{2} C_{2} O H, R_{6}=H$ $188 R_{1}=R_{4}=R_{6}=H, R_{2}=R_{7}=O H, R_{3}=G, R_{5}=O$ $189 R_{1}=R_{4}=R_{6}=H, R_{2}=R_{7}=O H, R_{3}=G, R_{5}=O A$ $194 R_{1}=R_{3}=R_{4}=H, R_{2}=R_{7}=O H, R_{5}=O F, R_{6}=O M$ $199 R_{1}=R_{3}=R_{4}=R_{6}=H, R_{2}=R_{7}=O H, R_{5}=O M e$ $200 R_{1}=A, R_{2}=R_{5}=R_{7}=O H, R_{3}=E, R_{4}=R_{6}=H$ $201 R_{1}=E, R_{2}=R_{5}=R_{7}=O H, R_{3}=A, R_{4}=R_{6}=H$
$202 R_{1}=A, R_{2}=R_{5}=R_{7}=O H, R_{3}=R_{4}=R_{6}=H$ $212 R_{1}=C_{2} H_{2} C_{2} \mathrm{OE}_{2} \mathrm{R}_{2}=\mathrm{R}_{5}=\mathrm{OH}, \mathrm{R}_{3}=\mathrm{R}_{4}=\mathrm{R}_{\mathrm{B}}=\mathrm{H}_{1} \mathrm{R}_{7}=\mathrm{OH}$ $213 R_{1}=R_{3}=A, R_{2}=R_{7}=O H, R_{4}=R_{5}=O M e, R_{6}=H$ $216 R_{1}=A, R_{2}=R_{7}=O H, R_{3}=R_{4}=R_{6}=H, R_{5}=O M e$ $217 R_{1}=A, R_{2}=R_{4}=R_{7}=O H, R_{3}=R_{6}=H, R_{5}=O M e$ $218 R_{1}=R_{3}=R_{4}=R_{6}=H, R_{2}=R_{5}=R_{7}=O H$ $219 R_{1}=R_{3}=R_{4}=R_{6}=H, R_{2}=R_{7}=O H, R_{5}=O F$ $220 R_{1}=R_{3}=R_{4}=R_{6}=H, R_{2}=O F, R_{5}=R_{7}=O H$
$221 R_{1}=R_{3}=R_{3} R_{2}=R_{4}=R_{5}=R_{7}=O H$ $222 R_{1}=R_{5}=H, R_{2}=R_{5}=R_{7}=O H, R_{3}=R_{4}=A$ $224 R_{1}=R_{4}=R_{6}=H, R_{2}=R_{5}=R_{7}=O H, R_{3}=A$ $225 R_{1}=R_{4}=A, R_{2}=R_{5}=R_{7}=O H, R_{3}=R_{6}=H$ $226 R_{1}=E, R_{2}=R_{5}=R_{7}=O H, R_{3}=R_{4}=R_{6}=H$ $227 R_{1}=A, R_{2}=R_{4}=R_{5}=R_{7}=O H, R_{3}=E, R_{6}=H$

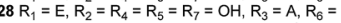
$230 R_{1}=R_{3}=R_{6}=H, R_{2}=R_{4}=R_{5}=R_{7}=O H$ $231 R_{1}=R_{3}=R_{6}=H, R_{2}=O R_{1}, R_{4}=R_{5}=R_{7}=O H$ $236 R_{1}=R_{3}=R_{6}=H \quad R_{2}=O M e, R_{4}=R_{5}=R_{7}=O H$ $237 R_{1}=R_{3}=R_{4}=R_{6}=H, R_{2}=O G, R_{5}=R_{7}=O H$ $241 R_{1}=R_{3}=R_{6}=H, R_{2}=R_{5}=R_{7}=O H, R_{4}=O M$ $243 \mathrm{R}_{1}=\mathrm{Me}, \mathrm{R}_{2}=\mathrm{R}_{7}=\mathrm{OH}, \mathrm{R}_{3}=\mathrm{G}, \mathrm{R}_{4}=\mathrm{R}_{6}=\mathrm{H}, \mathrm{R}_{5}=\mathrm{OE}$ $244 R_{1}=M e, R_{2}=R_{7}=O H, R_{3}=G, R_{4}=R_{6}=H_{1}, R_{5}=O A$ $246 R_{1}=R_{3}=R_{4}=R_{6}=H, R_{2}=R_{5}=O H, R_{7}=O M e$
251

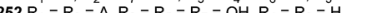
$254 R_{1}=R_{3}=A_{1} R_{2}=R_{4}=R_{5}=R_{7}=O H, R_{1}=H$ $255 R_{1}=A, R_{2}=R_{4}=R_{5}=R_{7}=O H, R_{3}=R_{8}=H$ $256 R_{1}=R_{3}=R_{4}=R_{6}=H, R_{2}=R_{5}=O M e, R_{7}=O H$ $257 R_{1}=R_{4}=R_{6}=H, R_{2}=R_{3}=R_{5}=R_{7}=O H$<smiles>CC(C)=CCc1c2c(c(O)c3c1OCC(c1ccc(O)cc1)C3=O)OC(C)(C)CO2</smiles>

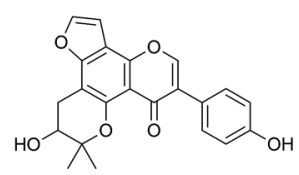

181

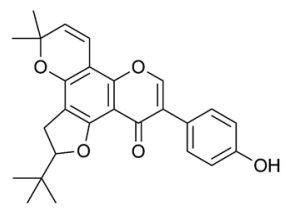

185

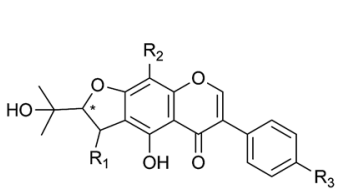
$203 R_{1}=R_{2}=R_{2}, R_{3}=O H$
$208 R_{1}=H, R_{2}=A, R_{3}=O H$ $208 R_{1}=H, R_{2}=A, R_{3}=O H$
$245(2 " S) R_{1}=R_{2}=O H, R_{3}=O M e$
$197 \mathrm{R}_{1}=\mathrm{OMe}, \mathrm{R}_{2}=\mathrm{H}, \mathrm{R}_{3}=\mathrm{OH}$<smiles>COCc1ccc(OC2OC(C)(C)C=Cc3c2c2c(c4occ(-c5ccc(O)cc5)c(=O)c34)C(O)C(O)C(C)(C)O2)cc1</smiles>

187

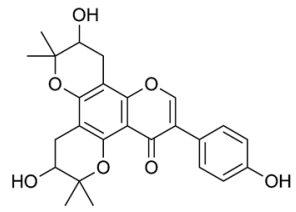

183
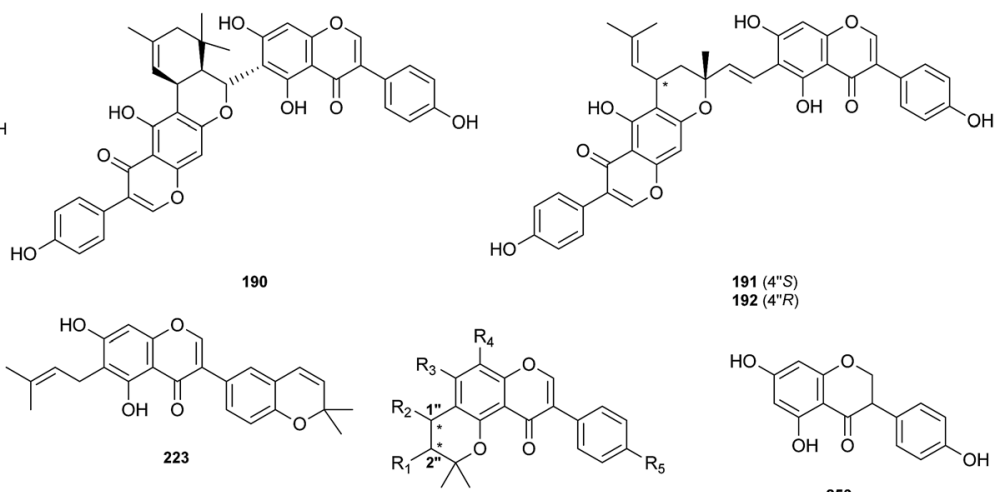

$182 \mathrm{R}_{1}=\mathrm{R}_{3}=\mathrm{OH}, \mathrm{R}_{2}=\mathrm{R}_{4}=\mathrm{H}, \mathrm{R}_{5}=\mathrm{OMe}$

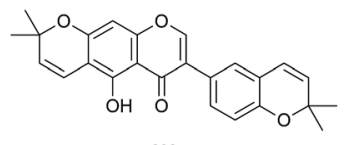

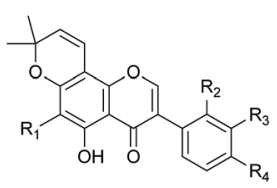

$195 R_{1}=R_{2}=H, R_{3}=R_{4}=O H$
$207 R_{1}=E, R_{2}=R_{3}=H, R_{4}=O H$ $207 R_{1}=E, R_{2}=R_{3}=H, R_{4}=O H$
$210 R_{1}=R_{2}=R_{3}=H, R_{4}=O H$ $211 R_{1}=R_{2}=R_{3}=H, R_{4}=O M e$ $233 R_{1}=A, R_{2}=R_{3}=R_{4}=O H$
$234 R_{1}=R_{3}=H, R_{2}=R_{4}=O H$ $229 R_{1}=A, R_{2}=R_{3}=H, R_{4}=O H$

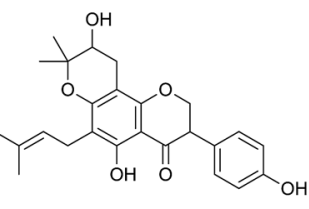

Fig. 2 (contd.)

via the regulation of NF- $\kappa$ B in LPS-stimulated RAW 264.7 cells. $^{34}$ The xanthone CTXA, as an effective inducer of heme oxygenase1 (HO-1), significantly inhibited the production of $\mathrm{PGE}_{2}, \mathrm{NO}$, TNF- $\alpha$, and IL-1 $\beta$ and increased the activity of HO in LPSstimulated RAW 264.7 macrophages. ${ }^{22}$ Moreover, CTXA could exert anti-soluble endothelial cell protein $\mathrm{C}$ receptor (antiSEPCR) shedding activity against vascular inflammation via inhibiting the expression of TNF- $\alpha$-converting enzyme induced by phorbol-12-myristate-13-acetate in endothelial cells. ${ }^{89}$ Cudraflavone B was not only a potent inhibitor of TNF- $\alpha$ by blocking the translocation of NF- $\mathrm{B}$ from the cytoplasm to the nucleus in macrophages derived from a THP-1 human monocytic leukemia cell line, but was also an inhibitor of COX-1 and COX-2 with higher selectivity toward COX-2, which suggested that it could be used as a lead for the development of nonsteroidal anti-inflammatory drugs. ${ }^{60}$
Allergic inflammation affects roughly one-quarter of people in the world. $.^{90} 5,7,3^{\prime}, 4^{\prime}$-Tetrahydroxy-6,8-diprenylisoflavone (254) not only interfered with the interaction between IgE and high-affinity IgE receptor (FceRI) and the expression of FceRI $\beta$ mRNA but also inhibited the redistribution of F-actin and downstream signalling by suppressing the activation of FceRImediated spleen tyrosine kinase in mast cells, which was suggestive of therapeutic potential for controlling mast cell activation in allergic processes. ${ }^{91}$ Treatment with the C. tricuspidata glycoprotein resulted in degranulation for allergic response ( $\beta$-hexosaminidase) and the activation of MAPK/ activator protein-1 (AP-1) and $\mathrm{NF}-\kappa \mathrm{B}$, as well as the expression of cytokines related to allergic inflammation (IL-4, IL-6, TNF- $\alpha$, IFN- $\gamma$, and IL-1 $\beta$ ), which are indirectly activated by bisphenol A or di(2-ethylhexyl) phthalate in HMC-1 and RBL-2H3 cells. ${ }^{33,92-96}$ 


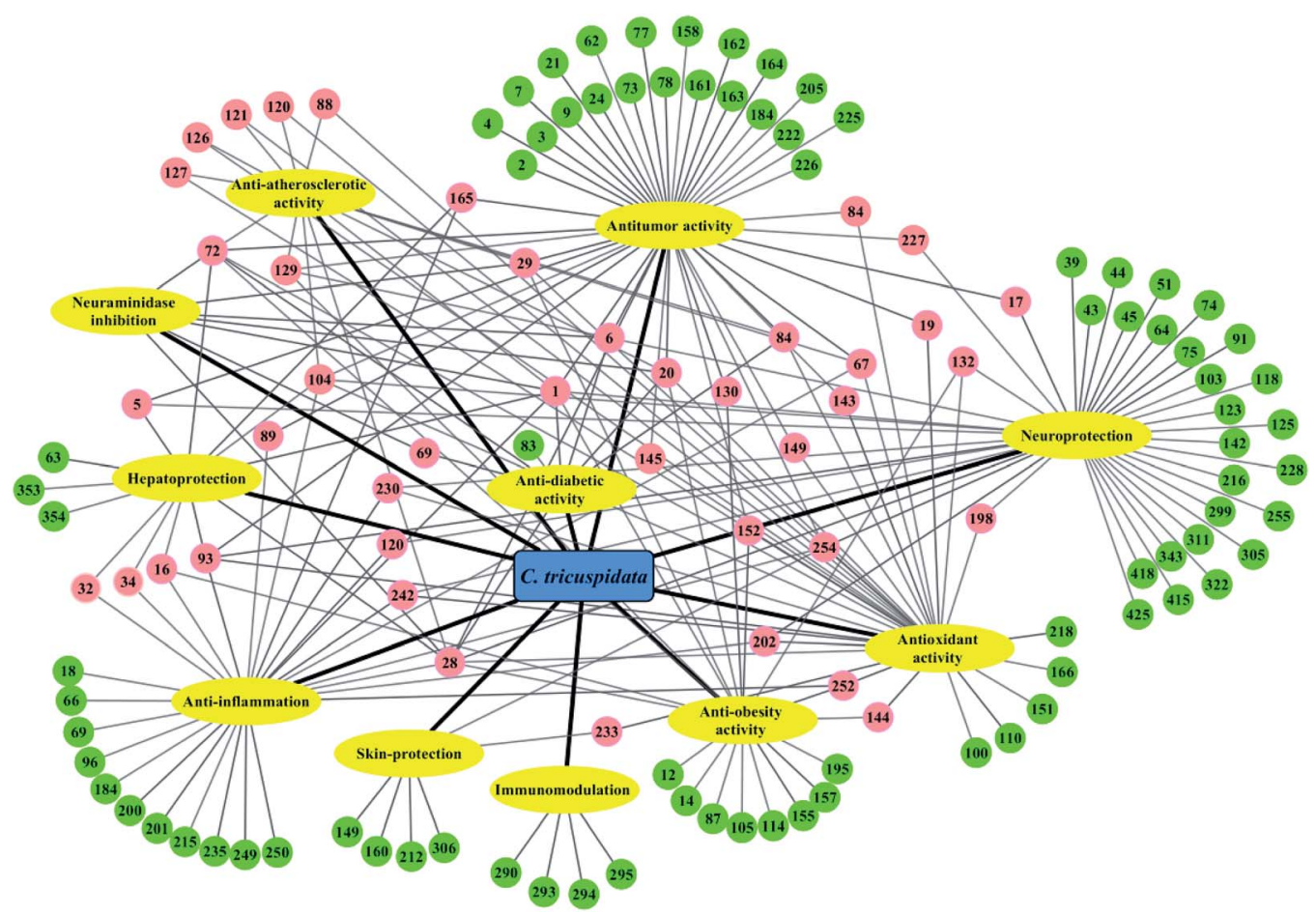

Fig. 3 Pharmacological activities of 124 active components from C. tricuspidata. The active compounds (circles) map ten pharmacological properties (yellow ovals). The pink circles represent multiple pharmacological properties. The green circles represent only one pharmacological property.

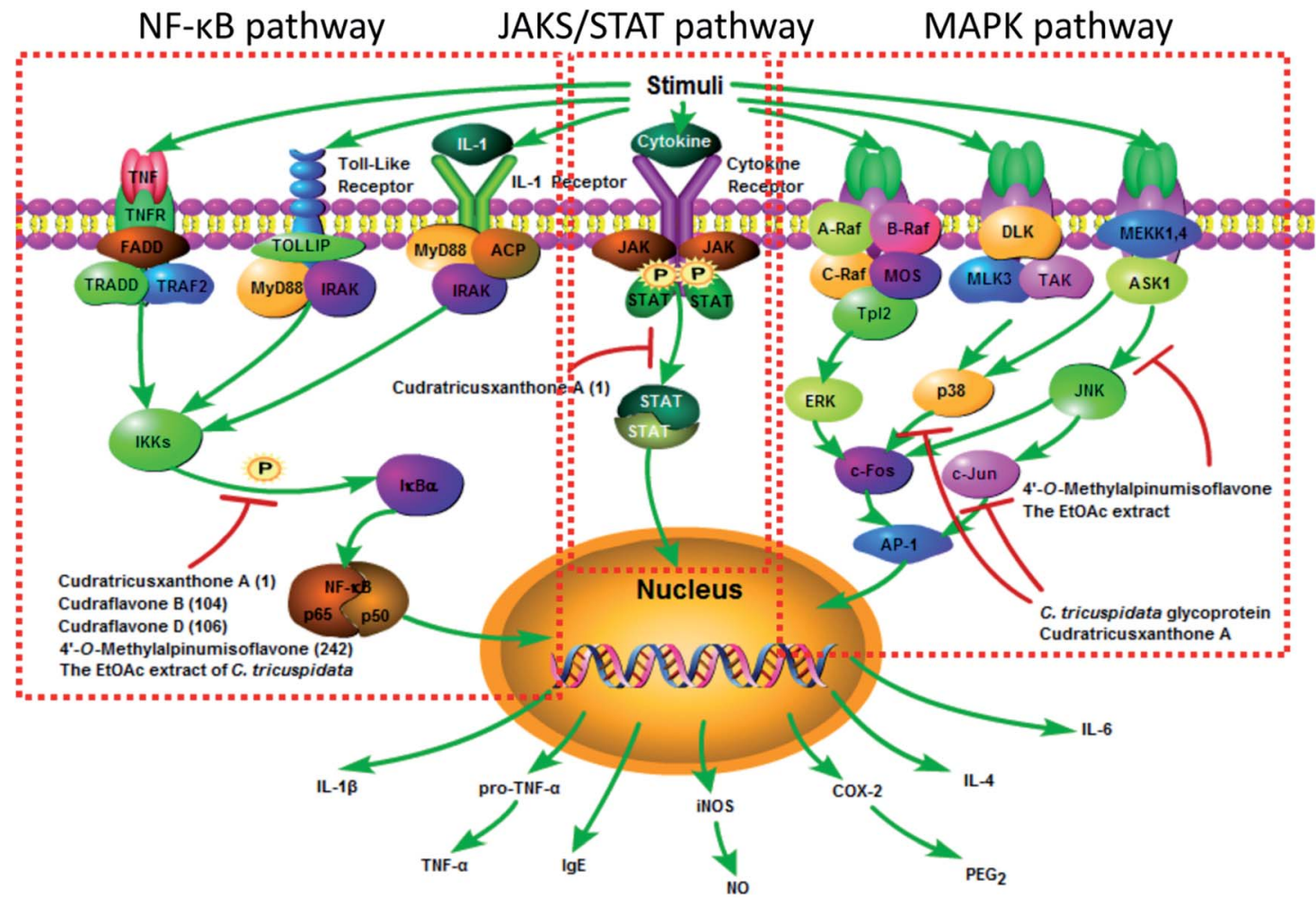

Fig. 4 Molecular mechanisms of anti-inflammatory extracts and compounds from C. tricuspidata. 
Table 2 Anti-inflammatory activities of prenylated xanthones and flavonoids against LPS-induced RAW 264.7 cells

\begin{tabular}{llllll}
\hline Compd & $\mathrm{IC}_{50}(\mu \mathrm{M})$ & Ref. & Compd & $\mathrm{IC}_{50}(\mu \mathrm{M})$ & Ref. \\
\hline $\mathbf{1 6}$ & 21.4 & 88 & $\mathbf{9 6}$ & 19.8 & 88 \\
$\mathbf{1 8}$ & 20.1 & 88 & $\mathbf{2 0 0}$ & 18.4 & 31 \\
$\mathbf{2 8}$ & 17.8 & 88 & $\mathbf{2 0 1}$ & 12.7 & 31 \\
$\mathbf{3 2}$ & 20.0 & 88 & $\mathbf{2 3 0}$ & 18.7 & 31 \\
$\mathbf{3 4}$ & 23.5 & 88 & $\mathbf{2 3 5}$ & 13.1 & 31 \\
$\mathbf{6 6}$ & 18.0 & 88 & $\mathbf{2 4 9}$ & 12.1 & 31 \\
$\mathbf{6 7}$ & 24.8 & 88 & $\mathbf{2 5 0}$ & 11.8 & 31 \\
$\mathbf{6 9}$ & 18.7 & 88 & $\mathbf{2 5 2}$ & 19.2 & 31 \\
$\mathbf{8 9}$ & 16.1 & 88 & & &
\end{tabular}

\subsection{Antioxidant activity}

Evidence has mounted that $C$. tricuspidata could act as an efficient free-radical scavenger and thus help the antioxidant defense system (Table 3). C. tricuspidata leaves, in comparison with other parts, exhibited the highest scavenging activities against the 1,1-diphenyl-2-picrylhydrazyl (DPPH) and 2,2'-azinobis(3-ethylbenzothiazoline-6-sulfonic acid) (ABTS) radicals and the highest ferric reducing/antioxidant power (FRAP), which was correlated with their high level of polyphenols $(73.60 \pm$ $0.28 \mathrm{mg} \mathrm{g}^{-1}$ ), in particular quercetin. ${ }^{97,98}$ It has been reported that $C$. tricuspidata leaves could produce more quercetin and kaempferol aglycones via Lactobacillus-mediated fermentation, which would increase antioxidant activities (DPPH and ABTS assays), and thereby could be developed as high-value-added food materials and functional foods. ${ }^{99-102}$ An aqueous extract of C. tricuspidata $\left(2 \mathrm{mg} \mathrm{mL}^{-1}\right)$ exhibited significant scavenging activities of $50.2 \%$ (ABTS) and $40.5 \%$ (FRAP), respectively. ${ }^{103}$ The antioxidant activity of C. tricuspidata fruits was revealed to change depending on the maturation stage and was positively associated with the contents of prenylflavonoids such as artocarpesin (100), alpinumisoflavone (198), 6-isopentenylgenistein (202), 4'-O-methylalpinumisoflavone and 6,8-diprenylgenistein. ${ }^{104}$ The prenyl group on the A-ring of isoflavone was a potent contributor against the ABTS radical system. ${ }^{105}$ The C. tricuspidata glycoprotein $\left(100 \mu \mathrm{g} \mathrm{mL} \mathrm{m}^{-1}\right)$ exhibited strong scavenging activities against DPPH, superoxide anions and hydroxyl radicals, with no pro-oxidant activity in vitro. ${ }^{34}$ It should be interesting to investigate the in vivo antioxidant potentials of these compounds for preventing various radical-mediated injuries in pathological situations.

\subsection{Antitumor activity}

During recent decades, C. tricuspidata has been demonstrated to possess promising antitumor and cytotoxic activities, and its cortices and root bark have been widely employed in TCM clinics for the treatment of cancer of the alimentary system, in particular gastric carcinoma., ${ }^{7,55}$ An EtOAc extract of

Table 3 Antioxidant activities of extracts and compounds of C. tricuspidata $^{a}$

\begin{tabular}{|c|c|c|c|c|}
\hline Sample & $\mathrm{DPPH}$ & ABTS & TBARS & Ref. \\
\hline The MeOH extract of root bark & $54.48 \mathrm{mg} \mathrm{mL}^{-1}$ & N.A & $15.13 \mathrm{mg} \mathrm{mL}^{-1}$ & 156 \\
\hline The ethyl ether fraction of $\mathrm{MeOH}$ extract of root bark & $30.78 \mathrm{mg} \mathrm{mL}^{-1}$ & N.A & $7.72 \mathrm{mg} \mathrm{mL}^{-1}$ & 156 \\
\hline The EtOAc fraction of $\mathrm{MeOH}$ extract of root bark & $20.32 \mathrm{mg} \mathrm{mL}^{-1}$ & N.A & $7.46 \mathrm{mg} \mathrm{mL}^{-1}$ & 156 \\
\hline Compd 28 & N.A & N.A & $3.8 \mu \mathrm{M}$ & 23 \\
\hline Compd 29 & N.A & N.A & $2.2 \mu \mathrm{M}$ & 23 \\
\hline Compd 67 & N.A & N.A & $0.8 \mu \mathrm{M}$ & 23 \\
\hline Compd 72 & N.A & N.A & $4.5 \mu \mathrm{M}$ & 23 \\
\hline Compd 84 & N.A & N.A & $12.6 \mu \mathrm{M}$ & 23 \\
\hline Compd 88 & N.A & N.A & $2.6 \mu \mathrm{M}$ & 23 \\
\hline Compd 126 & $4.04 \mu \mathrm{g} \mathrm{mL}^{-1}$ & N.A & $3.72 \mu \mathrm{g} \mathrm{mL}{ }^{-1}$ & 156 \\
\hline Compd 127 & $5.50 \mu \mathrm{g} \mathrm{mL}^{-1}$ & N.A & $3.71 \mu \mathrm{g} \mathrm{mL}^{-1}$ & 156 \\
\hline Compd 143 & $>300 \mu \mathrm{M}$ & $5.4 \mu \mathrm{M}$ & N.A & 157 \\
\hline Compd 144 & $>300 \mu \mathrm{M}$ & $6.0 \mu \mathrm{M}$ & N.A & 157 \\
\hline Compd 145 & N.A & N.A & $3 \mu \mathrm{g} \mathrm{mL}^{-1}$ & 30 \\
\hline Compd 149 & N.A & N.A & $10 \mu \mathrm{g} \mathrm{mL}^{-1}$ & 30 \\
\hline Compd 152 & $>300 \mu \mathrm{M}$ & $8.3 \mu \mathrm{M}$ & N.A & 157 \\
\hline Compd 166 & N.A & N.A & $6 \mu \mathrm{g} \mathrm{mL} L^{-1}$ & 30 \\
\hline Compd 230 & N.A & N.A & $3 \mu \mathrm{g} \mathrm{mL} L^{-1}$ & 30 \\
\hline Compd 254 & $>200 \mu \mathrm{M}$ & $16.3 \mu \mathrm{M}$ & N.A & 105 \\
\hline
\end{tabular}


C. tricuspidata stem bark displayed significant cytotoxicity against HL-60 cells, and the mechanism underlying its cytotoxicity may be due to apoptosis. ${ }^{106} \mathrm{~A} \mathrm{CHCl}_{3}$ extract of $C$. tricuspidata roots exhibited significant cytotoxicity against human gastric carcinoma cell lines (SGC-7901 and BGC-823). ${ }^{17}$ An $\mathrm{MeOH}$ extract of C. tricuspidata stems could induce the apoptosis of cervical cancer cells via the extrinsic pathway, as well as via the repression of human papillomavirus type-16 oncoproteins E6 and E7 and the alteration of p53 and p-pRb protein levels, instead of cytotoxicity. ${ }^{107}$ In nude mouse models of B16 melanoma and human SK-OV3 xenografted tumors, the tumor-inhibiting rates of the total flavonoids $\left(250 \mathrm{mg} \mathrm{kg}^{-1}\right)$ from C. tricuspidata were $50.54 \%$ and $46.38 \%$, respectively. ${ }^{108}$

Several compounds isolated from C. tricuspidata displayed considerable inhibitory activity against various tumor cells in

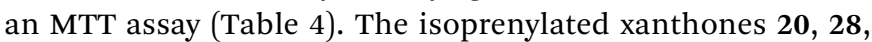
and 29 exhibited potent cytotoxic activity against HL-60 cells owing to apoptosis in a DNA fragmentation assay. ${ }^{56}$ Gericudranins A-E isolated from the stem bark of C. tricuspidata exhibited cytotoxicity against human tumor cell lines such as CRL1579 (skin), LOX-IMVI (skin), MOLT-4F (leukemia), KM12 (colon) and UO-31 (renal). ${ }^{\mathbf{1 6}, 58}$ The $p$-hydroxybenzyl moiety at C-6 was revealed to be essential for the cytotoxic activity. ${ }^{16,58}$ 2',5,7-Trihydroxy-4' , $^{\prime}$-(2,2-dimethylchromeno)-8-(3-hydroxy3-methylbutyl)flavanone (152) could inhibit the activity of topoisomerase $\mathrm{I}\left(\mathrm{IC}_{50}=1.0 \mathrm{mM}\right)$ and induce apoptotic cell death of U937 human leukemia cells, at least in part, via the inhibition of DNA topoisomerase I activity. ${ }^{109}$ Cudraflavanone A (129) inhibited mammalian topoisomerase I with an $\mathrm{IC}_{50}$ of $0.4 \mathrm{mM}$ and inhibited the activity of protein kinase $\mathrm{C}$ with an $\mathrm{IC}_{50}$ of $150 \mu \mathrm{M}^{{ }^{110}}$ Euchrestaflavanone B could inhibit the activity of protein kinase CKII with an $\mathrm{IC}_{50}$ of $78 \mu \mathrm{M}^{111}$ Cudraflavone B was demonstrated to be a lead for the development of a potential candidate for treating human oral squamous cell carcinoma cells via the activation of MAPK and $\mathrm{NF}-\mathrm{\kappa B}$, as well as the silent information regulator 1 (SIRT1) pathway. ${ }^{61}$ Cudraxanthone $\mathrm{H}(\mathbf{2 4})$ and isocudraxanthone $\mathrm{K}$ (62) exerted significant antiproliferative and apoptosisinducing effects in oral squamous cell carcinoma cells $\left(\mathrm{IC}_{50}\right.$ values of 14.31 and $17.91 \mu \mathrm{M}$ for HNSCC4 and 14.91 and 20.01 $\mu \mathrm{M}$ for HNSCC12 after treatment for $72 \mathrm{~h}$ ) via the NF- $\kappa \mathrm{B}$ and NIMA-interacting 1 pathways and mitochondrial death receptor, MAPK, NF- $\mathrm{kB}$, and HIF- $1 \alpha$ signalling pathways, respectively. ${ }^{112,113}$ Likewise, the cytotoxic effect of cudraflavone B was also documented against HNSCC4 cells $\left(\mathrm{IC}_{50}\right.$ of 18.3, 12.6, and $10.9 \mu \mathrm{M}$ after treatment for 24,48 , and $72 \mathrm{~h}$ ) and HNSCC12 cells $\left(\mathrm{IC}_{50}\right.$ of $19.5,12.0$, and $10.7 \mu \mathrm{M}$ after treatment for 24,48 , and $72 \mathrm{~h}) .{ }^{61}$ CTXA could suppress the migration and invasion of MCF-7 and MDA-MB-231 breast cancer cells by downregulating MMP-9 and induce apoptosis by activating the mitochondrial-associated apoptotic signalling pathway, which suggests that it may be a novel antitumor agent for breast cancer therapy. ${ }^{114}$ Cudratricusxanthone G could inhibit the proliferation, migration and invasion of SW620 human colorectal carcinoma cells instead of displaying cytotoxicity by targeting MMP-2, thereby regulating the activation of Rac1, Cdc42 and their downstream target AP-1. ${ }^{24}$ Notably, the chemical and biogenic synthesis and molecular modification of unique compounds isolated from C. tricuspidata have attracted attention. For example, from gericudranin A a series of derivatives were synthesized by structural modification, some of which exhibited strong cytotoxicity against several cancer cell lines such as SNB19, MOLT-4F, and K562 cells in a sulforhodamine B assay. ${ }^{115}$ It is suggested that more attention should be paid to the SAR and in vivo antitumor mechanisms of the antitumor constituents of $C$. tricuspidata.

\subsection{Hepatoprotective activity}

Liver disease remains one of the most serious health problems without satisfactory drugs. The $\mathrm{CHCl}_{3}$ fraction of an $\mathrm{MeOH}$ extract of C. tricuspidata root bark exhibited a significant hepatoprotective effect on tacrine-induced cytotoxicity in HepG2 cells. $^{26}$ CTXA, cudratricusxanthone $\mathrm{E}$, cudraxanthone $\mathrm{L}$ and macluraxanthone $\mathrm{B}$, which were isolated from the $\mathrm{CHCl}_{3}$ fraction, displayed the strongest hepatoprotective effects on tacrineinduced cytotoxicity in HepG2 cells at $10 \mu \mathrm{g} \mathrm{mL}{ }^{-1} .{ }^{26}$ Cudraflavone $\mathrm{B}$ and gericudranin $\mathrm{E}$ were further isolated from this $\mathrm{MeOH}$ extract and displayed significant protective effects against tacrine-induced cytotoxicity in $\mathrm{HepG} 2$ cells, with $\mathrm{EC}_{50}$ values of 37.39 and $39.87 \mu \mathrm{M}$, respectively. ${ }^{25}$ Cudracuspixanthone A (16) and cudracuspiphenones A (353) and B (354) exhibited moderate antiproliferative activity against HSC-T6 cells, with $\mathrm{IC}_{50}$ values of 9.7, 3.3, and $7.1 \mu \mathrm{M}$, respectively. ${ }^{116} \mathrm{It}$ was demonstrated that 1,1-dimethylallyl or 2,3,3-trimethyl-2,3dihydrofuran moieties in the xanthones played important roles for the inhibitory activity. ${ }^{116}$ The glycoprotein $(75 \mathrm{kDa})$ isolated from C. tricuspidata fruits was effective in preventing $\mathrm{CCl}_{4}$-induced liver damage in $\mathrm{A} / \mathrm{J}$ mice by significantly increasing the activities of superoxide dismutase, catalase, and glutathione peroxidase, as well as decreasing the production of TBARS, lactate dehydrogenase (LDH) and NO. ${ }^{35}$ These constituents might be preferred alternatives for liver disease, and in vivo assays are essential to ascertain their hepatoprotective role fully.

\subsection{Neuroprotective activity}

An aqueous extract of $C$. tricuspidata roots exhibited a stronger protective effect against neurotoxicity induced by oxidative stress than those of leaves, stems, and fruits, which was correlated with its high level of phenolic compounds, in particular kaempferol, myricetin (123) and quercetin. ${ }^{117}$ CTXA and cudraflavone B displayed significant neuroprotective activity against glutamate-induced neurotoxicity via the induction of HO-1 in HT22 mouse hippocampal cells. ${ }^{51,62}$ The neuroprotective effect of cudraflavone $\mathrm{B}$ was probably regulated by the phosphatidylinositol 3-kinase (PI3K)/AKT pathways. ${ }^{62}$

MAOs are responsible for the degradation of neurotransmitters including noradrenaline, dopamine, and 5-hydroxytryptamine in the central nervous system. ${ }^{118}$ The dichloromethane $\left(\mathrm{CH}_{2} \mathrm{Cl}_{2}\right)$ fraction of $C$. tricuspidata fruits was active in inhibiting mouse brain MAO, and gancaonin A (216), 
Table 4 Cytotoxic activities of extracts, xanthones and flavonoids against tumor cells

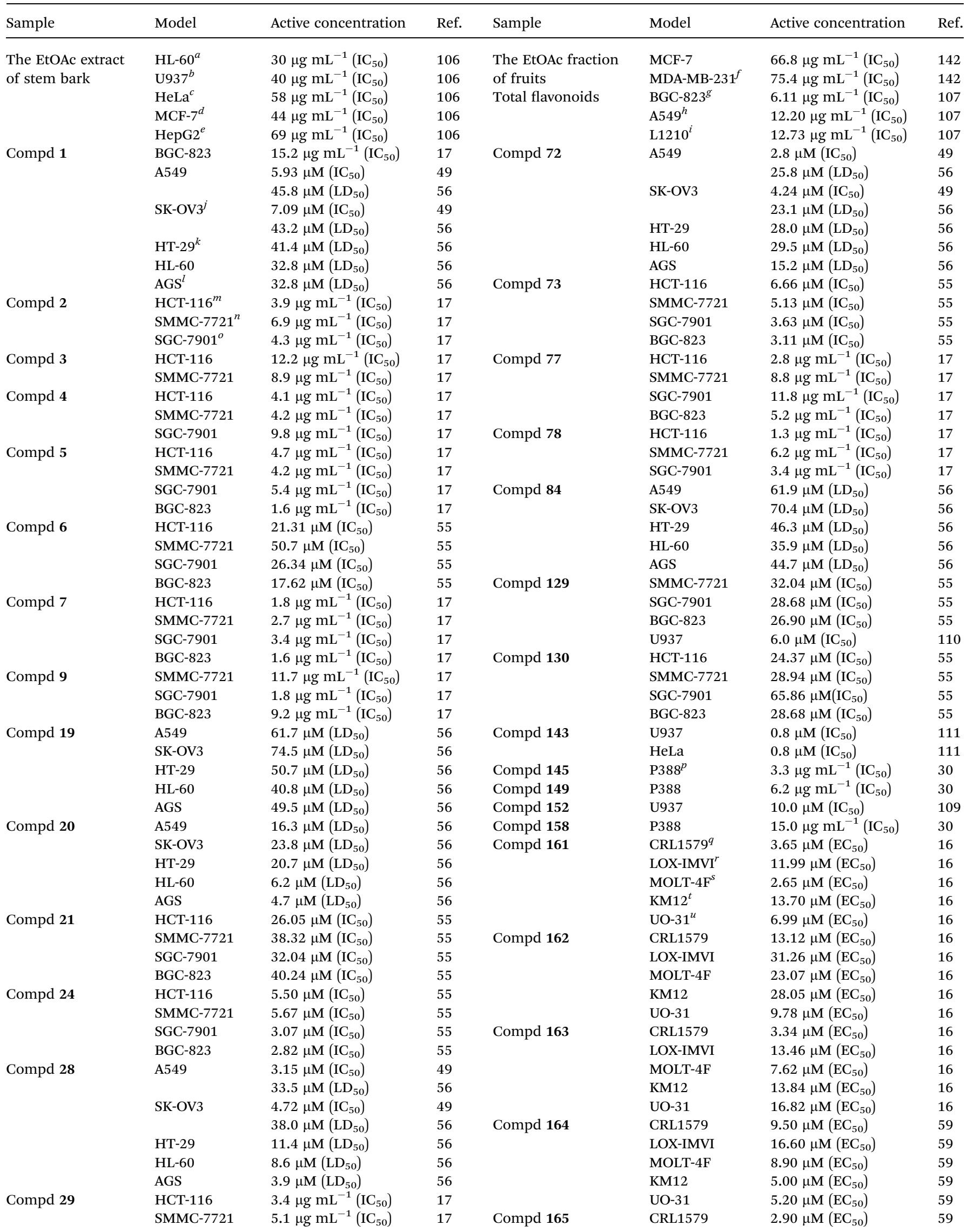


Table 4 (Contd.)

\begin{tabular}{|c|c|c|c|c|c|c|c|}
\hline Sample & Model & Active concentration & Ref. & Sample & Model & Active concentration & Ref. \\
\hline & SGC-7901 & $9.5 \mu \mathrm{g} \mathrm{mL} L^{-1}\left(\mathrm{IC}_{50}\right)$ & 17 & & LOX-IMVI & $12.50 \mu \mathrm{M}\left(\mathrm{EC}_{50}\right)$ & 59 \\
\hline & BGC-823 & $2.6 \mu \mathrm{g} \mathrm{mL}{ }^{-1}\left(\mathrm{IC}_{50}\right)$ & 17 & & MOLT-4F & $10.7 \mu \mathrm{M}\left(\mathrm{EC}_{50}\right)$ & 59 \\
\hline & SK-OV3 & $14.6 \mu \mathrm{M}\left(\mathrm{LD}_{50}\right)$ & 56 & & UO-31 & $7.60 \mu \mathrm{M}\left(\mathrm{EC}_{50}\right)$ & 59 \\
\hline & HT-29 & $12.1 \mu \mathrm{M}\left(\mathrm{LD}_{50}\right)$ & 56 & Compd 202 & HL-60 & $18.0 \mu \mathrm{M}\left(\mathrm{IC}_{50}\right)$ & 87 \\
\hline & HL-60 & $8.2 \mu \mathrm{M}\left(\mathrm{LD}_{50}\right)$ & 56 & Compd 205 & HL-60 & $4.3 \mu \mathrm{M}\left(\mathrm{IC}_{50}\right)$ & 87 \\
\hline & SK-OV3 & $71.3 \mu \mathrm{M}\left(\mathrm{LD}_{50}\right)$ & 56 & Compd 225 & HL-60 & $8.8 \mu \mathrm{M}\left(\mathrm{IC}_{50}\right)$ & 87 \\
\hline & HT-29 & $65.0 \mu \mathrm{M}\left(\mathrm{LD}_{50}\right)$ & 56 & Compd 226 & HL-60 & $10.1 \mu \mathrm{M}\left(\mathrm{IC}_{50}\right)$ & 87 \\
\hline & HL-60 & $45.2 \mu \mathrm{M}\left(\mathrm{LD}_{50}\right)$ & 56 & Compd 227 & HL-60 & $5.2 \mu \mathrm{M}\left(\mathrm{IC}_{50}\right)$ & 87 \\
\hline & AGS & $43.9 \mu \mathrm{M}\left(\mathrm{LD}_{50}\right)$ & 56 & Compd 246 & P388 & $0.18 \mu \mathrm{g} \mathrm{mL}-1\left(\mathrm{IC}_{50}\right)$ & 30 \\
\hline Compd 184 & HL-60 & $9.5 \mu \mathrm{M}\left(\mathrm{IC}_{50}\right)$ & 87 & Compd 254 & HL-60 & $4.3 \mu \mathrm{M}\left(\mathrm{IC}_{50}\right)$ & 87 \\
\hline
\end{tabular}

${ }^{a}$ HL-60 = promyelocytic leukemia cell line. ${ }^{b}$ U937 = human leukemia cell line. ${ }^{c}$ HeLa $=$ human carcinoma cell line. ${ }^{d}$ MCF-7 $=$ human breast cancer cell line. ${ }^{e}$ HepG2 = human hepatoma cell line. ${ }^{f}$ MDA-MB-231 = human breast cancer cell line. ${ }^{g}$ BGC-823 = stomach cancer cell line. ${ }^{h}$ A549 = lung carcinoma cell line. ${ }^{i}$ L1210 $=$ mouse leukemia cell line. ${ }^{j}$ SK-OV3 $=$ human ovarian cancer cell line. ${ }^{k}$ HT-29 $=$ human colon carcinoma cell line. ${ }^{l}$ AGS $=$ human lung cancer cell line. ${ }^{m}$ HCT-116 $=$ human colon carcinoma cell line. ${ }^{n}$ SMMC-7721 $=$ human hepatocellular carcinoma cell line. ${ }^{o}$ SGC-7901 $=$ human gastric cancer cell line. ${ }^{p}$ P388 $=$ mouse leukemia cell line. ${ }^{q}$ CRL1579 $=$ human skin cancer cell line. ${ }^{r}$ LOX-IMVI $=$ human melanoma cell line. ${ }^{s}$ MOLT-4F $=$ human leukemia cell line. ${ }^{t}$ KM12 $=$ human colon carcinoma cell line. ${ }^{u}$ UO-31 = human renal cell line.

4'-O-methylalpinumisoflavone, and alpinumisoflavone inhibited $\mathrm{MAO}$ in a concentration-dependent manner, with $\mathrm{IC}_{50}$ values of $19.4,23.9$, and $25.8 \mu \mathrm{M}$, respectively. Of these, gancaonin A exhibited a selective inhibitory effect against MAO-B $\left(\mathrm{IC}_{50}=0.8 \mu \mathrm{M}\right)$ in comparison with MAO-A $\left(\mathrm{IC}_{50}>800 \mu \mathrm{M}\right){ }^{118}$ CTXA, cudraflavanone A and cudraflavone $\mathrm{B}$ exhibited moderate inhibitory effects against mouse brain MAO, with $\mathrm{IC}_{50}$ values of $88.3,89.7$, and $80.0 \mu \mathrm{M}$, respectively. ${ }^{50}$

The neuroprotective potential of the flavonoids orobol (230), 6-prenylorobol (255) and 6,8-diprenylorobol was evaluated via enhancing the ubiquitin/proteasome-dependent degradation of $\alpha$-synuclein and synphilin-1 in SH-SY5Y human neuroblastoma cells induced by 6-hydroxydopamine (6-OHDA) (Table 5), which signified that they might be possible candidates for the treatment of neurodegenerative diseases. ${ }^{119}$ 5,7-Dihydroxychromone (426) could prevent 6-OHDA-induced oxidative stress and apoptosis in SH-SY5Y cells via the activation of the Nrf2/ARE

Table 5 Neuroprotective activity of compounds against 6-OHDAinduced SH-SY5Y cells

\begin{tabular}{lcllcl}
\hline Compd & $\mathrm{EC}_{50}(\mu \mathrm{M})$ & Ref. & Compd & $\mathrm{EC}_{50}(\mu \mathrm{M})$ & Ref. \\
\hline $\mathbf{1 7}$ & 4.5 & 27 & $\mathbf{1 4 2}$ & 9.1 & 145 \\
$\mathbf{2 8}$ & 8.2 & 27 & $\mathbf{2 2 7}$ & 15.2 & 119 \\
$\mathbf{3 9}$ & 7.2 & 27 & $\mathbf{2 2 8}$ & 18.5 & 119 \\
$\mathbf{4 3}$ & 16.6 & 27 & $\mathbf{2 3 0}$ & 6.4 & 119 \\
$\mathbf{4 4}$ & 2.4 & 27 & $\mathbf{2 5 4}$ & 10.1 & 119 \\
$\mathbf{4 5}$ & 2.2 & 27 & $\mathbf{2 5 5}$ & 4.5 & 119 \\
$\mathbf{5 1}$ & 0.8 & 27 & $\mathbf{3 0 5}$ & 9.2 & 145 \\
$\mathbf{6 4}$ & 3.0 & 27 & $\mathbf{3 1 1}$ & 8.0 & 145 \\
$\mathbf{7 4}$ & 15.5 & 27 & $\mathbf{3 2 2}$ & 12.9 & 145 \\
$\mathbf{7 5}$ & 0.7 & 27 & $\mathbf{3 4 3}$ & 6.2 & 145 \\
$\mathbf{9 1}$ & 2.3 & 27 & $\mathbf{4 1 6}$ & 11.2 & 168 \\
$\mathbf{9 7}$ & 5.1 & 27 & $\mathbf{4 1 9}$ & 30.2 & 145 \\
$\mathbf{1 0 3}$ & 15.5 & 145 & $\mathbf{4 2 6}$ & 1.9 & 145
\end{tabular}

signalling pathway and the overexpression of antioxidant enzymes, including $\mathrm{HO}-1, \mathrm{NAD}(\mathrm{P}) \mathrm{H}$ : quinone oxidoreductase and the glutamate-cysteine ligase catalytic subunit. ${ }^{120}$ In LPSstimulated BV2 mouse microglia, CTXA $\left(\mathrm{IC}_{50}=0.98 \mu \mathrm{M}\right)$ decreased the production of TNF- $\alpha$, IL-1 $\beta$, and IL-12, inhibited the phosphorylation and degradation of IкB- $\alpha$, and blocked the nuclear translocation of p50 and p65 by inhibiting the NF- $\kappa \mathrm{B}$ and MAPK pathways. ${ }^{121}$ Cudraflavanone D (132) could suppress the production of NO in LPS-induced BV2 microglial cells with an $\mathrm{IC}_{50}$ value of $6.28 \mu \mathrm{M}$ and exert anti-neuroinflammatory activity by targeting iNOS and COX-2 via the MAPK and NF- $\kappa \mathrm{B}$ pathways. ${ }^{1}$ Demethylsuberosin (299), as a potent proteasome activator, attenuated the 1-methyl-4-phenylpyridinium-induced dysfunction of the chymotrypsin-like and caspase-like activities of proteasomes in SH-SY5Y cells with $\mathrm{EC}_{50}$ values of $0.76 \mu \mathrm{M}$ and $0.82 \mu \mathrm{M}$, respectively, and protected SH-SY5Y cells against 1-methyl-4-phenylpyridinium-induced cell death, with an $\mathrm{EC}_{50}$ value of $0.17 \mu \mathrm{M} .{ }^{122} 4^{\prime}-O$-Methylalpinumisoflavone isolated from C. tricuspidata fruits exerted anti-neuroinflammatory effects against LPS-induced microglial activation in BV2 cells by decreasing NF- $\kappa \mathrm{B}$ signalling and the phosphorylation of MAPKs. ${ }^{123}$ The above results demonstrated that those compounds with neuroprotective activities could be considered as candidates for further research for therapeutic purposes into neurodegenerative diseases such as Parkinson's disease.

\subsection{Antiobesity activity}

Excess body weight and obesity are severe threats to public health worldwide. The leaves of $C$. tricuspidata, in comparison with other parts, exhibited the most pronounced inhibitory effect against pancreatic lipase (PL), which is a key enzyme for lipid absorption, with an $\mathrm{IC}_{50}$ value of $9.91 \mu \mathrm{g} \mathrm{mL} \mathrm{m}^{-1}$ in vitro, and were able to reduce plasma triacylglycerol levels and delay 
dietary fat absorption in vivo. ${ }^{18}$ The optimal conditions for the maximum PL-inhibiting activity and extraction yield of C. tricuspidata fruits were determined using response surface methodology to be an ethanol concentration of $74.5 \%$, a temperature of $61.9{ }^{\circ} \mathrm{C}$, and an extraction time of $13.5 \mathrm{~h} .{ }^{12}$ Flavonoids isolated from $C$. tricuspidata, namely, cudraflavanones A and D and 5,7,4'-trihydroxy-6,8-diprenylisoflavone, inhibited PL, with $\mathrm{IC}_{50}$ values of 9.0, 6.5, and $65.0 \mu \mathrm{M}$, respectively. ${ }^{12,124}$ Further SAR studies highlighted that the prenyl moiety and number and position of hydroxyl groups of the flavonoids seemed to affect the PL-inhibiting activity, which needs to be clarified using more derivatives. The PL-inhibiting activity of $C$. tricuspidata fruits has been proven to vary with their maturation stage. ${ }^{66}$ Unripe fruits of $C$. tricuspidata, in accordance with their higher content of total phenolic compounds and flavonoids, exhibited stronger PL-inhibiting activity in comparison to ripe fruits. ${ }^{66}$ In addition, an isoflavone, namely, cudracusisoflavone B (195), from unripe fruits exhibited strong PL-inhibiting activity, with an $\mathrm{IC}_{50}$ value of 16.8 $\mu \mathrm{M}$, in a non-competitive manner. ${ }^{66}$ Therefore, the maturation stage is an important factor for the efficacy, and unripe fruits appeared to be a good source of agents for the regulation of obesity. Protein-tyrosine phosphatases (PTP1B) are also important risk factors for obesity-related metabolic diseases. The leaves of $C$. tricuspidata displayed a strong inhibitory effect against PTP1B and substantially inhibited fat accumulation in 3T3-L1 cells in a dose-dependent manner. ${ }^{19}$ Xanthones and flavonoids isolated from the roots of $C$. tricuspidata, including CTXA, cudratricusxanthones L (12) and N (14), cudracuspixanthone A, cudraxanthones $\mathrm{D}, \mathrm{L}$, and $\mathrm{M}$, macluraxanthone $\mathrm{B}$, 1,6,7-trihydroxy-2-(1,1-dimethyl-2-propenyl)-3-

methoxyxanthone (87), cudraflavone C (105), kuwanon C (114), cudraflavanone D and euchrestaflavanone C, displayed a significant inhibitory activity against PTP1B in a dosedependent manner, with $\mathrm{IC}_{50}$ values ranging from 1.9 to 13.6 $\mu \mathrm{M} .{ }^{125}$ In comparison with flavonoids, prenylated xanthones displayed stronger PTP1B-inhibiting effects, which suggested that they may be promising agents for the future discovery of novel PTP1B inhibitors.

An aqueous extract of $C$. tricuspidata leaves that underwent fermentation mediated by lactic acid bacteria was proven to be beneficial for promoting osteogenic differentiation of osteoblastic cells and inhibiting fat accumulation in adipocytes. ${ }^{99}$ In diet-induced obesity (DIO) mice, this extract could decrease levels of aspartate aminotransferase, alanine aminotransferase, total fat mass, triglycerides, and blood glucose and was also found to promote the phosphorylation of IRS-1 and Akt in liver tissues and improve insulin secretion. ${ }^{19}$ Correspondingly, the leaves of $C$. tricuspidata could be used as materials to produce a functional food product with antiobesity effects. ${ }^{126}$ 6,8-Diprenylgenistein, which is a flavonoid isolated from C. tricuspidata, was proven to decrease body weight, epididymal fat and serum triglyceride levels in DIO mice. ${ }^{127}$ The underlying mechanism of this compound has been demonstrated, namely, that it could inhibit lipogenic genes by the regulation of transcription factors such as peroxisome proliferator-activated receptor $\gamma$ (PPAR $\gamma$ ) and CCAAT/enhancer-binding protein $\alpha(\mathrm{C} / \mathrm{EBP} \alpha)$ and hormones such as leptin and adiponectin. ${ }^{127}$ 6,8-Diprenylgenistein was also found to regulate acetyl-CoA carboxylase (ACC) and hydroxy-3-methylglutaryl coenzyme A reductase (HMGCR) by the activation of AMP-activated protein kinase (AMPK). ${ }^{127}$ Further investigation is warranted to determine whether their beneficial effects are associated with gut microbiota, which is a topic of recent and growing interest.

\subsection{Immunomodulatory effects}

Emerging evidence has suggested that $C$. tricuspidata is a potent immunomodulator. An aqueous extract of C. tricuspidata displayed potent adjuvant activity to enhance antigen-specific antibody responses and cellular immune responses against keyhole limpet hemocyanin. ${ }^{\mathbf{1 2 8}}$ In recent years, plant polysaccharides have emerged as an important class of bioactive natural products that are ideal therapeutic candidates for immunomodulatory functions with low toxicity. The in vitro immunomodulatory activities of the polysaccharides from $C$. tricuspidata roots were investigated in relation to the activation of mouse peritoneal macrophages. ${ }^{71,74}$ The results showed that the four water-soluble polysaccharides, namely, CTPS-1A, CTPS2B, CTPS-3A, and CTP-B1, could directly stimulate the proliferation of mouse splenocytes alone or in combination with concanavalin A or LPS within the concentration range of 6.25 to $100 \mu \mathrm{g} \mathrm{mL}{ }^{-1}$, in a comparable way to the immunomodulator lentinan. ${ }^{71,74}$ T-helper type 1 (Th1) and Th2 cytokines have been demonstrated to interact reciprocally to maintain a balanced immune network. The $C$. tricuspidata glycoprotein could prevent the development of immune diseases related to Th2 cell responses, such as autoimmune diseases, viral infections, and allergies. ${ }^{36,129}$ The precise mechanism of the differentiation of Th cells into Th1 or Th2 cells as induced by the $C$. tricuspidata glycoprotein remains to be elucidated.

\subsection{Antiatherosclerotic activity}

CTXA from C. tricuspidata was found to exert inhibitory effects on the synthesis and proliferation of DNA in vascular smooth muscle cells stimulated by platelet-derived growth factor (PDGF)-BB by suppressing the PDGF receptor $\beta$-chain and downregulating the Ras-Raf-MEK-ERK1/2 signalling pathways, and may serve as an antiatherosclerotic lead compound. ${ }^{\mathbf{8 0}}$ Likewise, cudraflavanone A was useful in the prevention of atherosclerosis or restenosis after angioplasty, and the molecular mechanism was found to be that it inhibited the PDGF-BBinduced growth of rat aortic smooth muscle cells via an Aktdependent pathway. ${ }^{81}$ In addition, cudraflavone $B$ was observed to inhibit the proliferation of rat aortic smooth muscle cells by inducing the expression of $\mathrm{p} 21^{\mathrm{cip} 1}$ and $\mathrm{p} 27^{\mathrm{kip} 1}$ and subsequent cell cycle arrest with a reduction in the phosphorylation of $\mathrm{pRb}$ at the G1-S phase, which suggests its therapeutic potential for treating cardiovascular disease. ${ }^{9}$ Low-density lipoprotein (LDL) has been known to play a crucial role in the development of atherosclerosis and hypercholesterolemia. ${ }^{\mathbf{1 3 0}}$ Many compounds isolated from $C$. tricuspidata have been confirmed to be effective in preventing the oxidation of LDL in a TBARS assay (Table 3). 


\subsection{Antimicrobial activity}

The essential oil of $C$. tricuspidata fruits was proven to be able to disrupt the membrane functions of both Gram-positive and Gram-negative bacteria, which led to its effective use as a natural antimicrobial agent to control food-borne pathogens in the food industry. The antibacterial activity of the essential oil was investigated against Bacillus cereus ATCC 13061, Staphylococcus aureus ATCC 12600, Listeria monocytogenes ATCC 7644, Salmonella typhimurium ATCC 43174 and Escherichia coli O157:H7 ATCC $43889 .^{76}$ The minimum inhibitory concentration (MIC) and minimum bactericidal concentration (MBC) of the essential oil were in the range of $250-500 \mu \mathrm{g} \mathrm{mL}^{-1}$ and $500-$ $1000 \mu \mathrm{g} \mathrm{mL}{ }^{-1}$, respectively. ${ }^{76}$ In addition, a methanolic extract of $C$. tricuspidata roots exhibited high antifungal activity against Gymnosporangium haraeanum Syd., Pyricularia oryzae Cav., Rhizoctonia solani Kühn, and Colletotrichum graminicola (Ces.) Wilson, with $\mathrm{EC}_{50}$ values of 803, 997, 981 and $930 \mu \mathrm{g} \mathrm{mL}{ }^{-1}$, respectively. ${ }^{11}$

\subsection{Skin protection}

Atopic dermatitis (AD) is a chronic inflammatory skin disease characterized by elevated immunoglobulin E (IgE) levels, mast cell infiltration and skin lesions including pruritus, erythema and eczema. ${ }^{131}$ An ethanolic extract of $C$. tricuspidata stems could be applied topically to decrease serum IgE levels and mast cell counts in the dermis of the skin in an AD-like NC/Nga mouse model induced by Dermatophagoides farinae extract. ${ }^{\mathbf{1 3 1}}$ Similarly, an aqueous extract of $C$. tricuspidata fruits was also found to inhibit the development of $\mathrm{AD}$-like skin lesions induced by repeated applications of $D$. farinae in sensitized NC/ Nga mice by reducing plasma concentrations of mouse thymus and activation-regulated chemokine (mTARC), histamine and IgE. ${ }^{\mathbf{1 3 2}}$ Nevertheless, the definite active compounds responsible for the anti-atopic dermatitis activity remain to be identified.

A methanolic extract of C. tricuspidata stems was demonstrated to prevent skin inflammation and skin aging via suppressing the solar ultraviolet-induced expression of COX-2. ${ }^{82}$ The EtOAc fraction $\left(\mathrm{IC}_{50}=24.4 \mathrm{ppm}\right)$ and the $n$-BuOH fraction $\left(\mathrm{IC}_{50}=\right.$ $88.3 \mathrm{ppm}$ ) of the $C$. tricuspidata stem extract could reduce the activity of tyrosinase and the melanin content in a concentrationdependent manner. ${ }^{79}$ It was found that the flavonoids steppogenin $\left(149, \mathrm{IC}_{50}=2.52 \mu \mathrm{M}\right)$ and trans-dihydromorin $\left(\mathbf{1 6 0}, \mathrm{IC}_{50}=\right.$ $21.54 \mu \mathrm{M})$ and the phenylpropanoid oxyresveratrol $\left(\mathrm{IC}_{50}=2.85\right.$ $\mu \mathrm{M}$ ) from the twigs of $C$. tricuspidata displayed potent inhibitory activities against mushroom tyrosinase and the melanogenesis process in melanocytes, which suggested their potential to be developed as skin-whitening agents in cosmetics and antibrowning agents in food. ${ }^{75}$ The tyrosinase-inhibiting activity of the flavonoids could be affected by the hydroxyl groups substituted at the 2- and 4-positions of the aromatic ring. ${ }^{75} \mathrm{Oxy}$ resveratrol and trans-dihydromorin, as hypopigmenting agents, could induce post-transcriptional degradation of microphthalmia-associated transcription factor (MITF), leading to significant decreases in the production of tyrosinase-related protein 1 (TRP-1) and tyrosinase-related protein 2 (TRP-2) in b16 and melan-a cells. ${ }^{133}$ Besides, 6,8-diprenylorobol and pomiferin (233) could inhibit the photooxidation of A2E, which is an important constituent of lipofuscin in the retinal pigment epithelium, in a dose-dependent manner. ${ }^{32}$ Collectively, these studies clearly showed that $C$. tricuspidata and the isolated bioactive compounds could be used as cosmeceutical materials and food constituents for the promotion of skin health.

\subsection{Antidiabetic activity}

Lee $e t a{ }^{20}{ }^{20}$ reported that the aqueous extract of $C$. tricuspidata leaves could significantly improve hepatic insulin resistance and hyperglycemia by controlling obesity-induced stress in the hepatic endoplasmic reticulum and inflammation in the liver of $\mathrm{db} / \mathrm{db}$ mice. Furthermore, an in vitro study demonstrated that both $C$. tricuspidata leaves and the isolated compound kaempferol could reduce hepatic insulin resistance by suppressing insulin receptor substrate signalling and the inflammatory response in HepG2 cells induced by endoplasmic reticulum stress. ${ }^{134}$ In addition, the $\alpha$-glucosidase-inhibiting activities of aqueous extracts of $C$. tricuspidata stems and roots depended on the harvesting time and climate. ${ }^{21} \mathrm{~A}$ root extract exerted potent inhibitory effects on $\alpha$-glucosidase activity, with $77 \%$ inhibition at a concentration of $300 \mu \mathrm{g} \mathrm{mL} \mathrm{m}^{-1}$, which signified that the root could serve as an antidiabetic biomaterial. ${ }^{21}$ Xanthones, including CTXA, cudratricusxanthone F, cudraxanthones D and $\mathrm{L}$, macluraxanthone B, 1,3,6,7-tetrahydroxy-2-(3-methylbut2-enyl)-8-(2-methylbut-3-en-2-yl)-9H-xanthen-9-one $\quad(83)$ and 1,3,7-trihydroxy-4-(1,1-dimethyl-2-propenyl)-5,6-(2,2-dimethylchromeno)xanthone (84), displayed inhibitory activities against $\alpha$-glucosidase, with $\mathrm{IC}_{50}$ values of $16.2-52.9 \mu \mathrm{M} .{ }^{52}$ CTXA was also proven to prevent the production of NO, the expression of iNOS, and the activation of JAK/STAT and NF- $\kappa \mathrm{B}$ in RINm5F cells induced by IL-1 $\beta$ and IFN- $\gamma$ and to inhibit the glucosestimulated secretion of insulin in pancreatic islets. ${ }^{135}$ The above results suggested that $C$. tricuspidata may be a promising therapeutic material in the treatment of diabetes.

\subsection{Others}

Besides the above pharmacological properties, other biological activities of $C$. tricuspidata have also been reported. An aqueous extract of $C$. tricuspidata stems could decrease systolic blood pressure in hypertension induced by $N^{\mathrm{G}}$-nitro-L-arginine methyl ester, in part by enhancing the generation of vascular NO/cGMP and the amelioration of renal functions. ${ }^{136}$ The anticoagulant activity of CTXA was investigated by Yoo et al., ${ }^{29}$ who revealed that CTXA could inhibit the generation of cell-based thrombin, activated factor X (FXa) and thrombin and exhibited thrombolytic activity by decreasing the ratio of plasminogen activator inhibitor type 1 (PAI-1) to tissue-type plasminogen activator ( $\mathrm{t}$ PA).

Park et $a l .{ }^{53}$ revealed that xanthones bearing 6,7 vicinal dihydroxy groups on the A ring, including CTXA, cudratricusxanthone $\mathrm{F}$, cudraxanthones $\mathrm{D}, \mathrm{L}$ and $\mathrm{M}$, macluraxanthone B, and 1,3,6,7-tetrahydroxy-2-(3-methylbut-2-enyl)-8-(2methylbut-3-en-2-yl)-9H-xanthen-9-one, displayed nanomolar inhibitory activity $\left(\mathrm{IC}_{50}: 80-270 \mathrm{nM}\right)$ against neuraminidase. Cudraflavanone A, which bears a C-8 hydrated prenyl group, 
also displayed high neuraminidase-inhibiting activity, with an $\mathrm{IC}_{50}$ of $380 \mathrm{nM}^{\mathbf{1 3 7}}$ This implied that these xanthones and flavonoids may be potential antiviral agents in the future.

The above descriptions indicated that many compounds have a variety of activities, in particular CTXA, which is a major and important component with a wide range of activities. Recently, pharmacokinetic studies of representative constituents of $C$. tricuspidata have also attracted attention. The in vitro metabolic profiling of CTXA in human liver microsomes has been recently investigated, which revealed that eight identified metabolites of CTXA were involved with cytochrome P450 enzymes (CYPs) and uridine 5'-diphospho-glucuronosyltransferase enzymes (UGTs). ${ }^{138}$ In a follow-up study, CTXA has been demonstrated to exhibit reversible competitive inhibition of CYP1A2 and CYP2C9 and non-competitive inhibition of CYP2C8 in human liver microsomes, which has begun to shed light on the in vivo metabolism of CTXA. ${ }^{139}$ Cudratricusxanthone $\mathrm{B}$, as another example, has also been investigated for its pharmacokinetics by a fast and sensitive HPLC-MS/ MS method, but its oral bioavailability (OB) remains unclear and merits future investigation. ${ }^{\mathbf{1 4 0}}$ Therefore, it is suggested that the pharmacokinetics of this plant should be studied systematically.

\section{Conclusions}

This review provides an up-to-date and comprehensive summary concerning the botany, traditional uses, phytochemistry and pharmacology of the traditional folk medicine C. tricuspidata. As a medicinal plant, C. tricuspidata has been used to treat rheumatism, bruising, scabies, hepatitis, jaundice, gonorrhea, dysmenorrhea and amenorrhea in East Asia for thousands of years. During the last few decades, C. tricuspidataderived extracts and compounds have attracted much attention for their promising biological activities, including antiinflammatory, antioxidant, antitumor, hepatoprotective, immunomodulatory, neuroprotective, antiobesity, antimicrobial, antiatherosclerotic, skin-protecting, and antidiabetic activities. Obviously, some pharmacological activities are not related to the traditional uses of this species but provide valuable hints for new areas of application. Xanthones and flavonoids are the two major classes of constituent that contribute either directly or indirectly to the biological effects of C. tricuspidata, followed by minor classes, including organic acids, polysaccharides, phenylpropanoids, and others. Findings and knowledge regarding the phytochemistry and pharmacology of C. tricuspidata have established a basis for further research on, and development of, this medicinal plant and its active components. Notably, the unique structures isolated from $C$. tricuspidata have aroused interest in research on the chemical and biogenic synthesis of these bioactive compounds that is suitable for large-scale preparation and molecular modification. This should be beneficial for the development and application of natural compounds from $C$. tricuspidata and their synthetic analogues. As a rich source of medicines and functional foods, quality control of $C$. tricuspidata is crucial to ensure both safety and efficacy. It is suggested that current advances, including mass spectrometry-based chemical profiling and DNA barcoding, should be used to authenticate, differentiate, and evaluate the quality of C. tricuspidata. Importantly, a common international criterion should be established with the ultimate goal of ensuring the effectiveness and safety and maximizing the medicinal benefits of $C$. tricuspidata.

As recent insights into the pharmacological mechanisms of C. tricuspidata are limited to in vitro bioassays of a limited number of molecules, it is essential and urgent to investigate the mechanisms of the bioactive extracts/isolates in appropriate animal models. To the best of our knowledge, few relevant data from clinical trials of $C$. tricuspidata (only in Chinese clinics) have been reported, and most clinical trials used a relatively small sample size and insufficient information. It is suggested that the efficacy of $C$. tricuspidata should be assessed in the future by combining its pharmacological effects, mechanisms of action and clinical applications. Detailed studies of the pharmacokinetics and toxicological properties and preclinical and clinical trials of $C$. tricuspidata are also eagerly awaited. More knowledge should be accumulated concerning the bioavailability, metabolism and toxicity of $C$. tricuspidata, which will be valuable for understanding its dosage efficacy and in vivo effects. It should be noted that the interaction between the bioactive constituents of $C$. tricuspidata and the human microbiota is an underappreciated aspect, as the gut microbiota plays a vital role in the pathogenesis and progression of obesity, diabetes and related metabolic disorders. ${ }^{\mathbf{1 4 1}}$ Current findings have demonstrated that $C$. tricuspidata may serve as a good source of prebiotics that promote the growth of probiotic bacteria and improve the antioxidant activity of dairy products, which is of great interest for the development of functional foods.

\section{Acknowledgements}

We would like to thank Drs Xue-qing Zhang (Ocean University of China), Cheng Qu (Nanjing University of Chinese Medicine) and master Miao-yin Zhang (The Johns Hopkins University) for critically reading a previous version of this manuscript. We apologize to authors whose relevant work was not included in this review owing to space constraints. This work was supported by the National High Technology Research and Development Program of China (863 Program) (No. 2013AA093001), The Scientific and Technological Innovation Project Financially Supported by Qingdao National Laboratory for Marine Science and Technology (No. 2015ASKJ02), and the Taishan Scholars Program, China.

\section{Notes and references}

1 D. C. Kim, C. S. Yoon, T. H. Quang, W. Ko, J. S. Kim, H. Oh and Y. C. Kim, Int. J. Mol. Sci., 2016, 17, 255.

2 B. S. Jung and M. K. Shin, Encyclopedia of Illustrated Korean Natural Drugs, Young Lim Press, Seoul, 1990.

3 L. Reich, Uncommon Fruits for Every Garden, Timber Press, Portland, 2004. 
4 T. Fujimoto, Y. Hano and T. Nomura, Planta Med., 1984, 50, 218-221.

5 Chinese Pharmacopoeia Commission, Pharmacopoeia of the People's Republic of China, China Medical Science and Technology Press, Beijing, 1997, vol. I, p. 467.

6 Nanjing University of Traditional Chinese Medicine, Dictionary of Chinese Herbal Medicine, Science and Technology Press, Shanghai, 2006.

7 Jiangsu New Medical College, Encyclopedia of Chinese Medicinal Substances, Shanghai People's Publisher, Shanghai, China, 1977.

8 H. H. Ding, D. H. Chen, L. F. Zhu, Q. Wu and X. H. Zhou, Zhongchengyao, 2001, 23, 151-152.

9 T. J. Kim, H. J. Han, Y. Lim, M. C. Song, J. Kim, J. T. Hong, H. S. Yoo, M. Y. Pyo, B. Y. Hwang, M. K. Lee and Y. P. Yun, J. Cardiovasc. Pharmacol., 2009, 53, 341-348.

10 S. Nam, H. W. Jang and T. Shibamoto, J. Agric. Food Chem., 2012, 60, 9097-9105.

11 Y. Z. Yu, Y. C. Deng, X. Qin, M. Zhang, L. F. Li and Z. W. Kong, Agrochemicals, 2009, 48, 224-226.

12 J. Y. Jeong, Y. H. Jo, K. Y. Lee, S. Do, B. Y. Hwang and M. K. Lee, Bioorg. Med. Chem. Lett., 2014, 24, 2329-2333.

13 Y. T. Hou, Tezhong Jingji Dongzhiwu, 2001, 7, 25.

14 O. K. Kim, D. E. Nam, W. J. Jun and J. M. Lee, J. Food Biochem., 2015, 39, 508-516.

15 S. H. Chang, E. J. Jung, D. G. Lim, B. Oyungerel, K. II Lim, E. Her, W. S. Choi, M. H. Jun, K. D. Choi, D. J. Han and S. C. Kim, J. Pharm. Pharmacol., 2008, 60, 1221-1226.

16 I. K. Lee, C. J. Kim, K. S. Song, H. M. Kim, H. Koshino, M. Uramoto and I. D. Yoo, Phytochemistry, 1996, 41, 213216.

17 Y. S. Zou, A. J. Hou, G. F. Zhu, Y. F. Chen, H. D. Sun and Q. S. Zhao, Bioorg. Med. Chem., 2004, 12, 1947-1953.

18 Y. S. Kim, Y. Lee, J. Kim, E. Sohn, C. S. Kim, Y. M. Lee, K. Jo, S. Shin, Y. Song, J. H. Kim and J. S. Kim, J. Evidence-Based Complementary Altern. Med., 2012, 277-293.

19 D. H. Kim, S. Lee, Y. W. Chung, B. M. Kim, H. Kim, K. Kim and K. M. Yang, BioMed Res. Int., 2016, 2016, 8432759.

20 O. K. Kim, D. E. Nam, W. J. Jun and J. M. Lee, Food Nutr. Res., 2015, 59, 29165.

21 H. U. Son and S. H. Lee, Biomed. Rep., 2013, 1, 624-628.

22 G. S. Jeong, D. S. Lee and Y. C. Kim, Int. Immunopharmacol., 2009, 9, 241-246.

23 K. H. Park, Y. D. Park, J. M. Han, K. R. Im, B. W. Lee, Y. Jeong, T. S. Jeong and W. S. Lee, Bioorg. Med. Chem. Lett., 2006, 16, 5580-5583.

24 L. Kuang, L. Wang, Q. Wang, Q. Zhao, B. Du, D. Li, J. Luo, M. Y. Liu, A. J. Hou and M. Qian, Biochem. Pharmacol., 2011, 81, 1192-1200.

25 R. B. An, D. H. Sohn and Y. C. Kim, Biol. Pharm. Bull., 2006, 29, 838-840.

26 Y. H. Tian, H. C. Kim, J. M. Cui and Y. C. Kim, Arch. Pharm. Res., 2005, 28, 44-48.

27 J. Kwon, N. T. Hiep, D. W. Kim, B. Y. Hwang, H. J. Lee, W. Mar and D. Lee, J. Nat. Prod., 2014, 77, 1893-1901.

28 N. T. Hiep, J. Kwon, D. W. Kim, B. Y. Hwang, H. J. Lee, W. Mar and D. Lee, Phytochemistry, 2015, 111, 141-148.
29 H. Yoo, S. K. Ku, W. Lee, S. Kwak, Y. D. Baek, B. W. Min, G. S. Jeong and J. S. Bae, Arch. Pharmacal Res., 2014, 37, 1069-1078.

30 I. K. Lee, K. S. Song, C. J. Kim, H. M. Kim, G. T. Oh and I. D. Yoo, Agric. Chem. Biotechnol., 1994, 37, 105-109.

31 X. H. Han, S. S. Hong, Q. Jin, D. Lee, H. K. Kim, J. Lee, S. H. Kwon, D. Lee, C. K. Lee, M. K. Lee and B. Y. Hwang, J. Nat. Prod., 2009, 72, 164-167.

32 G. M. Uddin, H. J. Lee, J. S. Jeon, D. Chung and Y. C. Kim, Nat. Prod. Sci., 2011, 17, 206-211.

33 J. Lee, S. J. Lee and K. T. Lim, Food Chem. Toxicol., 2012, 50, 2109-2117.

34 H. Y. Joo and K. T. Lim, Environ. Toxicol. Pharmacol., 2009, 27, 247-252.

35 H. Y. Joo and K. T. Lim, Korean J. Food Sci. Technol., 2009, 41, 93-99.

36 P. S. Oh and K. T. Lim, J. Appl. Toxicol., 2009, 29, 496-504.

37 Editorial Board of Flora of China, Flora of China, Science Publishing House, Beijing, 2003, vol. 5, pp. 35-36.

38 W. Y. Xiong, J. Z. Wang and T. D. Shi, Woody Medicine Plants of China, Shanghai: Shanghai Science and Education Press, Shanghai, 1993, pp. 85-88.

39 L. Shi, Asian J. Exp. Biol. Sci., 2010, 1, 311-314.

$40 \mathrm{M}$. Aleksandar, Journal of Mountain Agriculture on the Balkans, 2016, 19, 134-147.

41 H. J. Choi, C. T. Kim, Y. D. Min and M. J. Rang, J. Med. Plants Res., 2013, 14, 7-14.

42 Z. Q. Chen, Ben Cao Shi Yi, People's Medical Publishing House, Beijing, 1994, vol. II.

43 Z. S. Kou, Ben Cao Yan Yi, China Medical Science Press, Beijing, 2005.

44 Z. M. Mi, Ben Cao Hui Yan, Shanghai Science \& Technology Publishing House Press, Shanghai, 2005, p. 630.

45 B. D. Xiao, Lingnan Caiyao Lu, Guangdong Science Publishing House Press, Guangdong, 2009.

46 N. H. Li, Chinese Medicinal Herbs of Hong Kong, The Commercial Press, Hong Kong, 2002.

47 H. S. Guan and S. G. Wang, Chinese Marine Materia Medica, Science and Technology Press, Shanghai, China, 2009, p. 371.

48 Donguibogam Committee, Translated Donguibogam, Bubinmunwha Press, Seoul, 1999.

49 B. W. Lee, S. W. Gal, K. M. Park and K. H. Park, J. Nat. Prod., 2005, 68, 456-458.

50 J. H. Hwang, S. S. Hong, X. H. Han, J. S. Hwang, D. Lee, H. Lee, Y. P. Yun, Y. Kim, J. S. Ro and B. Y. Hwang, J. Nat. Prod., 2007, 70, 1207-1209.

51 G. S. Jeong, R. B. An, H. O. Pae, H. T. Chung, K. H. Yoon, D. G. Kang, H. S. Lee and Y. C. Kim, Planta Med., 2008, 74, 1368-1373.

52 E. J. Seo, M. J. Curtis-Long, B. W. Lee, H. Y. Kim, Y. B. Ryu, T. Jeong, W. S. Lee and K. H. Park, Bioorg. Med. Chem. Lett., 2007, 17, 6421-6424.

53 Y. B. Ryu, M. J. Curtis-Long, J. W. Lee, J. H. Kim, J. Y. Kim, K. Y. Kang, W. S. Lee and K. H. Park, Bioorg. Med. Chem., 2009, 17, 2744-2750.

54 P. J. O'Brien, Chem.-Biol. Interact., 1991, 80, 1-41. 
55 Y. S. Zou, A. J. Hou and G. F. Zhu, Chem. Biodiversity, 2005, 2, 131-138.

56 B. W. Lee, J. H. Lee, S. T. Lee, H. S. Lee, W. S. Lee, T. S. Jeong and K. H. Park, Bioorg. Med. Chem. Lett., 2005, 15, 55485552.

57 M. B. Wang, J. M. Huang and A. J. Hou, Fudan University Journal of Medical Sciences, 2006, 33, 559-562.

58 L. Chen, Y. Duan, C. Li, Y. Wang, X. Tong, Y. Dai and X. Yao, Magn. Reson. Chem., 2013, 51, 842-846.

59 I. K. Lee, C. J. Kim, K. S. Song, H. M. Kim, I. D. Yoo, H. Koshino, Y. Esumi and M. Uramoto, J. Nat. Prod., 1995, 58, 1614-1617.

60 J. Hošek, M. Bartos, S. Chudík, S. Dall'Acqua, G. Innocenti, M. Kartal, L. Kokoška, P. Kollăr, Z. Kutil, P. Landa, R. Marek, V. Závalová, M. Žemlička and K. Šmejkal, J. Nat. Prod., 2011, 74, 614-619.

61 H. J. Lee, Q. S. Auh, Y. M. Lee, S. K. Kang, S. W. Chang, D. S. Lee, Y. C. Kim and E. C. Kim, Planta Med., 2013, 79, 1298-1306.

62 D. S. Lee, W. Ko, D. C. Kim, Y. C. Kim and G. S. Jeong, Molecules, 2014, 19, 10818-10831.

63 B. W. Lee, N. S. Kang and K. H. Park, J. Korean Soc. Appl. Biol. Chem., 2004, 47, 270-273.

64 Y. Z. Yin, R. D. Chen, D. W. Zhang, L. R. Qiao, J. H. Li, R. S. Wang, X. Liu, L. Yang, D. Xie, J. H. Zou, C. M. Wang and J. G. Dai, J. Mol. Catal. B: Enzym., 2013, 89, 28-34.

65 R. S. Wang, R. D. Chen, J. H. Li, X. Liu, K. B. Xie, D. W. Chen, Y. Z. Yin, X. Y. Tao, D. Xie, J. H. Zou, L. Yang and J. G. Dai, J. Biol. Chem., 2014, 289, 35815-35825.

66 Y. H. Jo, S. B. Kim, Q. Liu, S. G. Do, B. Y. Hwang and M. K. Lee, PLoS One, 2017, 12, e0172069.

67 X. X. Huang, H. F. Lai and Z. W. Li, Her. Med., 2013, 32, 664666.

68 B. Li, M. Wang, Y. N. Tan, M. M. Tong and Y. J. Zhai, Zhongguo Zhongyao Zazhi, 2013, 38, 167-170.

69 J. S. Jeon, S. M. Kim, H. J. Lee, B. H. Um, H. K. Kim and C. Y. Kim, J. Liq. Chromatogr. Relat. Technol., 2012, 35, 1607-1615.

70 G. T. Jung, I. O. Ju, S. R. Choi, D. H. You and J. J. Noh, Korean Journal of Food Preservation, 2013, 20, 330-335.

71 L. Shi and Y. Fu, Acta Biochim. Biophys. Sin., 2011, 43, 418424.

72 L. Shi, K. Chen, Q. Dong, J. Fang and K. Ding, Front. Chem. China, 2008, 3, 209-212.

73 L. Shi, Y. L. Fu and K. S. Chen, Fitoterapia, 2007, 78, 298301.

74 L. Shi, Q. Dong and K. Ding, Food Chem., 2014, 152, 291296.

75 Z. P. Zheng, H. Y. Tan, J. Chen and M. Wang, Fitoterapia, 2013, 84, 242-247.

76 V. K. Bajpai, A. Sharma and K. H. Baek, Food Control, 2013, 32, 582-590.

77 E. N. Novruzov and U. M. Agamirov, Chem. Nat. Compd., 2002, 38, 468-469.

78 E. J. Cho, T. Yokozawa, D. Y. Rhyu, S. C. Kim, N. Shibahara and J. C. Park, Phytomedicine, 2003, 10, 544-551.
79 H. S. Han, S. Y. Kim, D. J. Lim and W. K. Whang, Asian Journal of Beauty and Cosmetology, 2016, 14, 317-328.

80 T. J. Kim, H. J. Han, S. S. Hong, J. H. Hwang, B. Y. Hawang, H. S. Yoo, Y. R. Jin, J. J. Lee, J. Y. Yu, K. H. Lee, B. W. Kang and Y. P. Yun, Biol. Pharm. Bull., 2007, 30, 805-809.

81 H. J. Han, T. J. Kim, Y. R. Jin, S. S. Hong, J. H. Hwang, B. Y. Hwang, K. H. Lee, T. K. Park and Y. P. Yun, Planta Med., 2007, 73, 1163-1168.

82 J. E. Kim and K. W. Lee, Int. J. Mol. Sci., 2015, 16, 2509625107.

83 G. Yang, K. Lee, M. Lee, I. Ham and H. Y. Choi, BMC Complementary Altern. Med., 2012, 12, 250.

84 W. G. Seo, H. O. Pae, G. S. Oh, K. Y. Chai, Y. G. Yun, T. O. Kwon and H. T. Chung, Gen. Pharmacol. Vasc. Syst., 2000, 35, 21-28.

85 E. G. Lee, H. J. Yun, S. I. Lee and W. H. Yoo, Korean J. Intern. Med., 2010, 25, 93-100.

86 E. G. Lee, S. Lee, H. J. Chae, S. J. Park, Y. C. Lee and W. H. Yoo, Biol. Res., 2010, 43, 225-231.

87 H. L. T. Anh, D. T. Tuan, D. T. Trang, B. H. Tai, N. X. Nhiem, P. H. Yen, P. V. Kiem, C. V. Minh, T. M. Duc, H. K. Kang, Y. C. Kim and Y. H. Kim, J. Asian Nat. Prod. Res., 2016, 1-9. 88 Y. H. Jo, S. B. Kim, Q. Liu, B. Y. Hwang and M. K. Lee, Arch. Pharm., 2017, 350, e1600263.

89 S. K. Ku, M. S. Han, G. S. Jeong and J. S. Bae, Anim. Cells Syst., 2014, 18, 9-16.

90 S. J. Galli, M. Tsai and A. M. Piliponsky, Nature, 2008, 454, 445-454.

91 T. Lee, J. Kwon, D. Lee and W. Mar, J. Agric. Food Chem., 2015, 63, 5459-5467.

92 J. U. Shim and K. T. Lim, Inflammation, 2009, 32, 211-217.

$93 \mathrm{~J}$. Lee and K. T. Lim, Naunyn-Schmiedeberg's Arch. Pharmacol., 2010, 382, 51-61.

94 C. H. Park and K. T. Lim, Immunol. Invest., 2010, 39, 171185.

95 P. S. Oh and K. T. Lim, J. Cell. Biochem., 2010, 109, 124-131. 96 P. S. Oh, K. Lim and K. T. Lim, Cell Biochem. Funct., 2010, 28, 352-359.

97 C. H. Jeong, G. N. Choi, J. H. Kim, J. H. Kwak, H. J. Heo, K. H. Shim, B. R. Cho, Y. Bae and J. S. Choi, Prev. Nutr. Food Sci., 2009, 14, 283-289.

98 J. Y. Kim, J. H. Chung, I. Hwang, Y. S. Kwan, J. K. Chai, K. H. Lee, T. H. Han and J. H. Moon, Korean J. Hortic. Sci. Technol., 2009, 27, 489-496.

99 Y. Lee, J. Oh, H. Lee, N. K. Lee, D. Y. Jeong and Y. S. Jeong, Biotechnol. Bioprocess Eng., 2015, 20, 861-870.

100 Y. Lee, J. Oh and Y. S. Jeong, Food Sci. Biotechnol., 2015, 24, 1817-1821.

101 N. S. Oh, J. Y. Lee, S. Oh, J. Y. Joung, S. G. Kim, Y. K. Shin, K. W. Lee, S. H. Kim and Y. Kim, J. Dairy Sci., 2016, 99, 1-12.

102 N. S. Oh, J. Y. Lee, J. Y. Joung, K. S. Kim, Y. K. Shin, K. W. Lee, S. H. Kim, S. Oh and Y. Kim, Appl. Microbiol. Biotechnol., 2016, 100, 5919-5932.

103 S. H. Nile and D. H. Kim, Nat. Prod. Commun., 2015, 10, 1839-1842.

104 G. R. Shin, S. Lee, S. Lee, S. Do, E. Shin and C. H. Lee, Ind. Crops Prod., 2015, 70, 322-331. 
105 J. H. Lee, B. W. Lee, J. H. Kim, W. D. Seo, K. C. Jang and K. H. Park, J. Appl. Biol. Chem., 2005, 48, 193-197.

106 W. G. Seo, H. O. Pae, G. S. Oh, K. Y. Chai, Y. G. Yun, H. T. Chung, K. K. Jang and T. O. Kwon, Am. J. Chin. Med., 2001, 29, 313-320.

107 S. B. Kwon, M. J. Kim, J. M. Yang, H. P. Lee, J. T. Hong, H. S. Jeong, E. S. Kim and D. Y. Yoon, PLoS One, 2016, 11, e0150235.

108 Z. Zhang, H. J. Wu, N. H. Pi, T. Zhang, W. Y. Yuan, A. J. Hou and J. H. Liu, World Clin. Drugs, 2009, 30, 601-605.

109 Y. H. Rho, S. H. Yoon, E. K. Kim, J. Y. Kang, B. W. Lee, K. H. Park and Y. S. Bae, Nat. Prod. Res., 2007, 21, 616-624.

110 Y. H. Rho, B. W. Lee, K. H. Park and Y. S. Bae, Anti-Cancer Drugs, 2007, 18, 1023-1028.

111 S. H. Kim, S. H. Yoon, B. W. Lee, K. H. Park, Y. H. Kim and Y. S. Bae, Oncol. Res., 2005, 15, 327-332.

112 H. J. Lee, S. S. Jue, S. K. Kang, W. J. Bae, Y. C. Kim and E. C. Kim, Am. J. Chin. Med., 2015, 43, 1-14.

113 M. R. Shin, H. J. Lee, S. K. Kang, Q. S. Auh, Y. M. Lee, Y. C. Kim and E. C. Kim, BioMed Res. Int., 2014, 2014, 934691.

114 S. M. Jeon, D. S. Lee and G. S. Jeong, J. Ethnopharmacol., 2016, 194, 57-62.

115 Y. J. Choi, H. M. Kim and H. D. Kim, Arch. Pharmacal Res., 2009, 32, 59-63.

116 Y. H. Jo, B. Shin, Q. Liu, K. Y. Lee, D. C. Oh, B. Y. Hwang and M. K. Lee, J. Nat. Prod., 2014, 77, 2361-2366.

117 C. H. Jeong, G. N. Choi, J. H. Kim, J. H. Kwak, H. R. Jeong, D. O. Kim and H. J. Heo, Food Sci. Biotechnol., 2010, 19, 1113-1117.

118 X. H. Han, S. S. Hong, J. S. Hwang, S. H. Jeong, J. H. Jeong, J. H. Hwang, M. H. Lee, M. K. Lee, D. Lee, J. S. Ro and B. Y. Hwang, Arch. Pharmacal Res., 2005, 28, 1324-1327.

119 D. W. Kim, J. Kwon, S. J. Sim, D. Lee and W. Mar, J. Funct. Foods, 2017, 29, 104-114.

120 D. W. Kim, K. T. Lee, J. Kwon, H. J. Lee, D. Lee and W. Mar, Life Sci., 2015, 130, 25-30.

121 C. S. Yoon, D. C. Kim, T. H. Quang, J. Seo, D. G. Kang, H. S. Lee, H. Oh and Y. C. Kim, Molecules, 2016, 21, 1-12.

122 B. H. Kim, J. Kwon, D. Lee and W. Ma, Planta Med. Lett., 2015, 2, e15-e18.

123 J. Y. Lim, B. Y. Hwang, K. W. Hwang and S. Y. Park, Phytother. Res., 2012, 26, 1948-1956.

124 Y. H. Jo, S. B. Kim, Q. Liu, J. W. Lee, B. Y. Hwang and M. K. Lee, Bioorg. Med. Chem. Lett., 2015, 25, 3455-3457.

125 T. H. Quang, N. T. T. Ngan, C. S. Yoon, K. H. Cho, D. G. Kang, H. S. Lee, Y. C. Kim and H. Oh, Molecules, 2015, 20, 11173-11183.

126 D. H. Suh, E. S. Jung, H. M. Park, S. H. Kim, S. Lee, Y. H. Jo, M. K. Lee, G. Jung, S. G. Do and C. H. Lee, PLoS One, 2016, 11, e0149022.

127 Y. H. Jo, K. M. Choi, Q. Liu, S. B. Kim, H. J. Ji, M. Kim, S. K. Shin, S. G. Do, E. Shin, G. Jung, H. S. Yoo, B. Y. Hwang and M. K. Lee, Nutrients, 2015, 7, 10480-10490.

128 K. B. Lee, K. S. Song, E. H. Moon, J. Lee and Y. C. Yoo, J. Korean Soc. Appl. Biol. Chem., 2009, 52, 234-240.
129 P. S. Oh and K. T. Lim, Naunyn-Schmiedeberg's Arch. Pharmacol., 2009, 380, 115-124.

130 W. Palinski, M. E. Rosenfeld, S. Ylä-Herttuala, G. C. Gurtner, S. S. Socher, S. W. Butler, S. Parthasarathy, T. E. Carew, D. Steinberg and J. L. Witztum, Proc. Natl. Acad. Sci. U. S. A., 1989, 86, 1372-1376.

131 Y. S. Park, S. H. Kim, S. Y. Kim, G. M. Koh, J. H. Suh and J. S. Kang, Pharmacol. Pharm., 2016, 7, 358-367.

132 H. Lee, H. Ha, J. K. Lee, C. Seo, N. Lee, D. Jung, S. Park and H. K. Shin, Phytother. Res., 2012, 26, 594-599.

133 S. T. Hu, Z. P. Zheng, X. C. Zhang, F. Chen and M. F. Wang, J. Funct. Foods, 2015, 13, 375-383.

134 O. K. Kim, W. J. Jun and J. M. Lee, Nutrients, 2016, 8, 60. 135 D. S. Lee and G. S. Jeong, Int. Immunopharmacol., 2014, 21, 26-33.

136 D. G. Kang, T. Y. Hur, G. M. Lee, H. Oh, T. O. Kwon, E. J. Sohn and H. S. Lee, Life Sci., 2002, 70, 2599-2609.

137 Y. B. Ryu, M. J. Curtis-Long, J. W. Lee, H. W. Ryu, J. Y. Kim, W. S. Lee and K. H. Park, Bioorg. Med. Chem. Lett., 2009, 19, 4912-4915.

138 J. Sim, E. Choi, G. S. Jeong and S. Lee, Biopharm. Drug Dispos., 2015, 36, 325-336.

139 J. Sim, E. Choi, Y. M. Lee, G. S. Jeong and S. Lee, Food Chem. Toxicol., 2015, 81, 171-175.

140 N. H. Pi, J. Chen, J. M. Huang and A. J. Hou, J. Chromatogr. B: Anal. Technol. Biomed. Life Sci., 2010, 878, 1953-1958.

141 J. Xu, H. B. Chen and S. L. Li, Med. Res. Rev., 2016, 1-46.

142 C. T. Cao, C. L. Sun, W. B. Bai, J. X. Sun, G. Q. Li, S. Y. Ou and Y. Yang, Shipin Kexue, 2015, 36, 48-51.

143 C. Lei, C. C. Liu, E. H. Pi and A. J. Hou, Helv. Chim. Acta, 2014, 97, 1683-1688.

144 T. Nomura, Y. Hano and T. Fujimoto, Heterocycles, 1983, 20, 213-218.

145 J. Kwon, N. T. Hiep, D. W. Kim, S. Hong, Y. Guo, B. Y. Hwang, H. J. Lee, W. Mar and D. Lee, J. Nat. Prod., 2016, 79, 1938-1951.

146 Y. Hano, Y. Matsumoto, J. Y. Sun and T. Nomura, Planta Med., 1990, 56, 399-402.

147 Y. Hano, Y. Matsumoto, J. Y. Sun and T. Nomura, Planta Med., 1990, 56, 478-481.

148 Y. Hano, Y. Matsumoto, K. Shinohara, J. Y. Sun and T. Nomura, Planta Med., 1991, 57, 172-175.

149 Z. P. Zheng, J. Y. Liang and L. H. Hu, J. Integr. Plant Biol., 2006, 48, 996-1000.

150 T. Fujimoto, Y. Hano, T. Nomura and J. Uzawa, Planta Med., 1984, 50, 161-163.

151 Y. Hano, Y. Matsumoto, K. Shinohara, J. Y. Sun and T. Nomura, Heterocycles, 1990, 31, 1339-1344.

152 W. C. Wu, Y. J. Zhai and Z. Y. Li, Zhongyaocai, 2010, 33, 913915.

153 Y. Z. Yin, R. S. Wang, R. D. Chen, L. R. Qiao, L. Yang, C. M. Wang and J. G. Dai, Zhongguo Zhongyao Zazhi, 2012, 37, 3734-3737.

154 H. S. Young, J. H. Park, H. J. Park and J. S. Choi, Arch. Pharmacal Res., 1989, 12, 39-41.

155 G. M. Zhang, X. Y. Xu and J. F. Xi, Zhongchengyao, 2008, 30, 771-773. 
156 Y. J. Lee, S. Kim, S. J. Lee, I. Ham and W. K. Whang, Arch. Pharmacal Res., 2009, 32, 195-200.

157 B. W. Lee, J. H. Lee, S. W. Gal, Y. H. Moon and K. H. Park, Biosci., Biotechnol., Biochem., 2006, 70, 427-432.

158 T. Fujimoto and T. Nomura, Planta Med., 1985, 51, 190-193.

159 W. G. Shan, L. L. Shi, Y. M. Ying, X. R. Hou and Z. J. Zhan, J. Chem. Res., 2013, 37, 285-286.

160 Y. Guan, Z. Yin, L. Guo, X. Huang, W. Ye and W. Shen, Zhongguo Zhongyao Zazhi, 2009, 34, 1108-1110.

161 A. K. Ibrahim, A. A. Mohamed and T. Mukul, World J. Pharm. Res., 2014, 3, 981-997.

162 Y. Kang, J. U. Choi, E. A. Lee and H. R. Park, Food Sci. Biotechnol., 2013, 22, 1449-1452.
163 H. J. Lee, S. T. Cho, T. G. Lee, U. J. Kim, E. C. Ma and D. H. Lee, Repub. Korean Kongkae Taeho Kongbo, 2015, 5, 33.

164 C. P. Li, X. J. Chang, L. Fang, J. B. Yao, R. W. Wang, Z. J. Zhan, Y. M. Ying and W. G. Shan, Chem. Nat. Compd., 2016, 52, 202-204.

165 A. P. Wang, M. C. Liu and S. J. Yang, Chin. J. Exp. Tradit. Med. Formulae, 2011, 17, 113-115.

166 C. H. Miu, Z. B. Gu and G. J. Yang, Zhongchengyao, 2002, 24, 211-212.

167 Y. H. Zhang, W. W. Ren and S. W. Wan, Yiyao Gongye, 1980, $3,15$.

168 N. T. Hiep, J. Kwon, D. W. Kim, S. Hong, Y. Guo, B. Y. Hwang, N. Kim, W. Mar and D. Lee, Tetrahedron, 2017, 73, 2747-2759. 